\title{
On $n$-superharmonic functions and some geometric applications
}

\author{
Shiguang $\mathrm{Ma}^{1} \cdot$ Jie Qing ${ }^{2}$
}

Received: 16 June 2020 / Accepted: 31 July 2021 / Published online: 22 September 2021

(c) The Author(s) 2021

\begin{abstract}
In this paper we study asymptotic behaviors of $n$-superharmonic functions at singularity using the Wolff potential and capacity estimates in nonlinear potential theory. Our results are inspired by and extend [6] of Arsove-Huber and [63] of Taliaferro in 2 dimensions. To study $n$-superharmonic functions we use a new notion of thinness in terms of $n$-capacity motivated by a type of Wiener criterion in [6]. To extend [63], we employ the Adams-MoserTrudinger's type inequality for the Wolff potential, which is inspired by the inequality used in [15] of Brezis-Merle. For geometric applications, we study the asymptotic end behaviors of complete conformally flat manifolds as well as complete properly embedded hypersurfaces in hyperbolic space. These geometric applications seem to elevate the importance of $n$-Laplace equations and make a closer tie to the classic analysis developed in conformal geometry in general dimensions.
\end{abstract}

\section{Introduction}

In this paper we will develop some understanding of isolated singularities of $n$-superharmonic functions in general dimensions and apply it to study some geometric problems. Recall that the $n$-Laplace operator

$$
\Delta_{n} u=\operatorname{div}\left(|\nabla u|^{n-2} \nabla u\right)
$$

is a quasilinear, possibly degenerate, elliptic operator that agrees with the Laplace operator in 2 dimensions.

Communicated by A. Chang.

S. Ma: The author is supported by NSFC 11571185 and NSFC 11871283

J. Qing: The author is partially supported by NSF DMS-1608782.

$凶 \quad$ Jie Qing

qing@ucsc.edu

Shiguang Ma

msgdyx8741@nankai.edu.cn

1 Department of Mathematics, Nankai University, Tianjin, China

2 Department of Mathematics, University of California, Santa Cruz, CA 95064, USA 
The theory of $n$-Laplace equations is as fundamental as that of classic Laplace equations since it is also in the center of the interplay of several important fields of mathematics including calculus of variations, partial differential equations, nonlinear potential theory, and mathematical physics. The theory of $n$-Laplace equations is more general as well as more challenging, because the principle of superposition is no longer available. We would like to develop higher dimensional extensions to what has been done for the theory of subharmonic functions in $[6,37,63]$ (references therein) regarding asymptotic behavior and their applications in differential geometry. Our research in this paper seems to elevate the importance of $n$-Laplace equations and makes a closer tie to the classic analysis developed in conformal geometry.

The first goal for us is to study the behavior of $n$-superharmonic functions at a point. The first main theorem in general dimensions is inspired by and extends the work of Arsove-Huber in [6, Theorem 1.3].

Theorem 1.1 Let $w$ be a nonnegative lower semi-continuous function that is $n$-superharmonic in $B(0,2) \subset \mathbb{R}^{n}$ and

$$
-\Delta_{n} w=\mu \geq 0
$$

for a Radon measure $\mu \geq 0$. Then there is a set $E \subset \mathbb{R}^{n}$, which is $n$-thin at the origin, such that

$$
\lim _{x \notin E \text { and }|x| \rightarrow 0} \frac{w(x)}{\log \frac{1}{|x|}}=\liminf _{|x| \rightarrow 0} \frac{w(x)}{\log \frac{1}{|x|}}=m \geq 0
$$

and

$$
w(x) \geq m \log \frac{1}{|x|}-C \text { for } x \in B(0,1) \backslash\{0\} \text { and some } C .
$$

Moreover, if $w \in C^{2}(B(0,2) \backslash\{0\})$ and $\left(B(0,2) \backslash\{0\}, e^{2 w}|d x|^{2}\right)$ is complete at the origin, then $m \geq 1$.

The definition of $n$-superharmonic functions is given in Definition 2.3. The definition of $n$ thinness is given in Definition 3.1, which is inspired by and extends the definition of thinness in [6] (see the discussion on the comparison of different notions of thinness in Sect. 2.2). The proof of Theorem 1.1 combines the blow-down argument from [49] and the nonlinear potential theory $[2,39,48,51,52]$ for $n$-Laplace equations, particularly the use of the Wolff potential and $n$-capacity estimates.

The proof of Theorem 1.1 consists of four major steps. The first is to use nonlinear potential theory, particularly [48, Theorem 1.6 and Lemma 3.9] on the Wolff potential and $n$-capacity estimates [cf. (3.9)], to show that the blow-down quotient

$$
w_{r}(\xi)=\frac{w(r \xi)}{\log \frac{1}{|r|}}
$$

is bounded outside a subset $\hat{E}$ that is $n$-thin. The second step is to use a cut-off technique from [27] to modify and cut off the unbounded part in order to take sequential limit for the blow-down quotients as $r \rightarrow 0$. Based on the Liouville Theorem of [39,55,59], one knows that the sequential limits are all constant. In the third step, we use the comparison principle (cf. [64, Lemma 3.1] and [49,50]) to conclude that all sequential limits have to be the same as $m$ in the Theorem 1.1. In the final step, based on the uniqueness of sequential limits, we re-run the proof in the first step to extract a subset $E$ that is $n$-thin and finish the proof of 
Theorem 1.1. The first run of the argument in the first step is to get bounds; while the second run is to get uniform convergence. It is essential and very interesting to see how the classic Paul du Bois-Reymond Theorem (cf. [57] and [16, (5) Page 40]) for infinite series helps to re-enforce the argument in the first run in applying [48, Theorem 1.6 and Lemma 3.9] [cf. (3.31)] to get the uniform convergence.

Our second goal is to eliminate nontrivial $n$-thin subsets $E$ in Theorem 1.1. This theorem in general dimensions is inspired by and extends the work of Taliaferro in [61-63].

Theorem 1.2 Let $w \in C^{2}(B(0,2) \backslash\{0\})$ be nonnegative and satisfy

$$
-\Delta_{n} w=f(x, w, \nabla w)
$$

in $B(0,2) \backslash\{0\} \subset \mathbb{R}^{n}$ and that

$$
\lim _{x \rightarrow 0} w(x)=+\infty
$$

where $f$ satisfies the critical growth condition

$$
0 \leq f(x, w, \nabla w) \leq C|\nabla w|^{n-2} e^{2 w}
$$

for some fixed constant $C$. Then

$$
\lim _{x \rightarrow 0} \frac{w(x)}{\log \frac{1}{|x|}}=m \geq 0
$$

and

$$
w(x) \geq m \log \frac{1}{|x|}-L \text { for } x \in B(0,1) \backslash\{0\}
$$

for some constant $L$.

The essential ingredient in the proof of Theorem 1.2 is a type of Brezis-Merle inequality

$$
\int_{\Omega} \exp \left(\frac{n(1-\delta) W_{1, n}^{\mu_{f}}(x, D)}{\|f\|_{L^{1}(\Omega)}^{\frac{1}{n-1}}}\right) d x \leq \frac{c(n) 2^{2 n+1}|B(0, D)|}{\delta^{n+1}}+2^{n}|\Omega| .
$$

for the Wolff potential

$$
W_{1, n}^{\mu_{f}}(x, D)=\int_{0}^{D} \mu_{f}(B(x, s))^{\frac{1}{n-1}} \frac{d s}{s}
$$

with

$$
\mu_{f}(A)=\int_{A \cap \Omega} f(x) d x
$$

induced by a nonnegative function in $L^{1}(\Omega)$, where $D$ is the diameter of $\Omega$ and $\delta \in(0,1)$. Equation (1.7) is stated in Proposition 4.1 and extends the inequality discovered by BrezisMerle in 2 dimensions (cf. $[15,32,44]$ and references therein). This Adams-Moser-Trudinger inequality (1.7) for the Wolff potential helps control any possible concentration and rule out any possible nontrivial $n$-thin subset $E$ in Theorem 1.1. As stated in Remark 4.1, the critical growth condition may be described as

$$
0 \leq f(x, w, \nabla w) \leq C|\nabla w|^{p} e^{\alpha w}
$$


for any $0<p<n$ and $\alpha>0$ to be more general. Our proof of Theorem 1.2 is a streamlined one from [61-63] with the help of the Adams-Moser-Trudinger inequality (1.7) for the Wolff potential.

As applications we first want to study asymptotic behavior at the end of complete locally conformally flat manifolds with nonnegative Ricci. After the classification theorems of [18, 67], we want to focus on complete metrics $e^{2 \phi}|d x|^{2}$ on $\mathbb{R}^{n}$. One may calculate and find that

$$
-\Delta_{n} \phi=\operatorname{Ric}_{g}\left(\nabla^{g} \phi\right)|\nabla \phi|^{n-2} e^{2 \phi},
$$

where $\operatorname{Ric}_{g}(\nabla \phi)$ is the Ricci curvature of the metric $g=e^{2 \phi}|d x|^{2}$ in $\nabla^{g} \phi$ direction. Equation (1.9) is clearly a generalization of Gauss curvature equations in higher dimensions.

Theorem 1.3 Suppose that $\left(\mathbb{R}^{n}, e^{2 \phi}|d x|^{2}\right)$ is complete with nonnegative Ricci, where $\phi$ is a smooth function. Then there is a subset $E \subset \mathbb{R}^{n}$, which is $n$-thin at infinity, such that

$$
\lim _{x \notin E \text { and } x \rightarrow \infty} \frac{\phi(x)}{\log \frac{1}{|x|}}=\liminf _{x \rightarrow \infty} \frac{\phi(x)}{\log \frac{1}{|x|}}=m
$$

and

$$
\phi(x) \geq m \log \frac{1}{|x|}-L
$$

for some constant $L$, where $m \in[0,1]$ and

$$
m^{n-1}=\frac{1}{w_{n-1}} \int_{\mathbb{R}^{n}} \operatorname{Ric}_{g}\left(\nabla^{g} \phi\right)|\nabla \phi|^{n-2} e^{2 \phi} d x .
$$

Moreover,

- $m=0$ if and only if $g=e^{2 \phi}|d x|^{2}$ is flat;

- if Ric ${ }_{g}$ is bounded, then

$$
\lim _{x \rightarrow \infty} \frac{\phi(x)}{\log \frac{1}{|x|}}=\liminf _{x \rightarrow \infty} \frac{\phi(x)}{\log \frac{1}{|x|}}=m .
$$

This theorem gives some precise description of asymptotic end behavior. More importantly it also includes a rigidity result that does not assume Ricci is bounded. The rigidity result in this theorem should be compared with [25, Theorem 0.3$]$ and $[7,18,23]$. It is particularly desirable to compare the blow-down approaches here and those in $[7,18,25]$. The proof appeals to Theorems 1.1 and 1.2. But it is not straightforward at all to calculate $m$ and derive the rigidity, especially when Ricci is not assumed to be bounded. Our argument relies on the natural and desirable construction of exhausting family of domains to perform integrations (please see $\Omega_{\varepsilon, t}^{ \pm}$in the proof of Theorem 5.2 in Sect. 5).

Our second application is to study the asymptotic behavior at the end of properly embedded complete hypersurfaces with nonnegative Ricci curvature in hyperbolic space. It was shown in [12, Main Theorem] that such hypersurfaces have at most two ends, and are equidistant hypersurfaces if with two ends. Based on Theorem 1.1, we are able to improve the theorems on asymptotic at infinity in $[3,4]$, where the hypersurfaces are assumed to have nonnegative sectional curvature.

Theorem 1.4 Suppose that $\Sigma^{n}$ is a properly embedded, complete hypersurface with nonnegative Ricci curvature and one single end in hyperbolic space $\mathbb{H}^{n+1}$. Then it is a global graph 
of $\rho=\rho(x)$ in Busemann coordinates and it is asymptotically rotationally symmetric in the sense that there is a number $m \in[0,1]$ such that

$$
m \log |x|+o(\log |x|) \leq \rho(x) \leq m \log |x|+C
$$

as $x \rightarrow \infty$ in $\mathbb{R}^{n}$. Moreover, $m=0$ implies that the hypersurface is a horosphere. In any case, the hypersurface $\Sigma$ always stays inside a horosphere and is supported by some equidistant hypersurface at infinity.

The proof follows from the one in $[3,4]$, and in fact is simpler than the one in $[3,4]$, because of Theorem 1.1. The use of $n$-subharmonic functions is more suitable than the use of subharmonic functions restricted to each 2-plane [cf. [3,4]]. To eliminate any nontrivial $n$-thin set $E$ in Theorem 1.1 in these cases, we use the strict convexity of the hypersurfaces, where the strong $n$-capacity lower bound estimate of Gehring (cf. [56, Lemma 1.4 page 212] and [33, Theorem 4]) is used sharply.

The rest of the paper is organized as follows: In Sect. 2, we present definitions and basic facts that are useful. We describe what have been done in 2 dimensions to motivate our study in this paper. We also explain the opportunity for the use of $n$-superharmonic functions in geometric problems. In Sect. 3, we define $n$-thinness by $n$-capacity and prove Theorem 1.1. In Sect. 4, we establish the Adams-Moser-Trudinger inequality for the Wolff potential and prove Theorem 1.2. In Sect. 5, we introduce the classification of complete locally conformally flat manifolds with nonnegative Ricci curvature and prove Theorem 1.3. Finally, in Sect. 6, we recall the classification of complete properly embedded hypersurfaces with nonnegative Ricci curvature and prove Theorem 1.4.

\section{Preliminaries and background}

In this section, after adopting the definitions of $n$-harmonic functions and $n$-superharmonic functions from [48, Section 2], we present a review of what has been done in 2 dimensions to motivate what we want to do in general dimensions. Then we introduce some background and tools from the theory of quasilinear elliptic equations and nonlinear potential theory that are useful to us. We also introduce the geometric problems that we expect to use $n$-superharmonic functions to study in this paper.

\subsection{Definitions of $n$-superharmonic functions}

We first discuss definitions of $n$-superharmonic functions first to clear any possible confusions caused by terminology. Let us recall the definitions of $n$-superharmonic functions from [51, Definition 2.5 and 2.12] and [48, Section 2].

Definition 2.1 ([51, Definition 2.5]) For a domain $\Omega \subset \mathbb{R}^{n}$, a function $u \in W_{\text {loc }}^{1, n}(\Omega)$ is said to be weakly $n$-harmonic in $\Omega$ if

$$
\int|\nabla u|^{n-2} \nabla u \nabla \phi=0
$$

for all $\phi \in C_{c}^{\infty}(\Omega)$. A weak $n$-harmonic function $u \in W_{\text {loc }}^{1, n}(\Omega)$ is said to be $n$-harmonic if it is continuous in $\Omega$.

We know from [51, Theorem 2.19] that any weak $n$-harmonic function always has a continuous representive and therefore $n$-harmonic. For further regularity of $n$-harmonic functions 
we refer readers to [51] and references therein. For the definitions of $n$-superharmonic functions, we first recall

Definition 2.2 ([51, Definition 2.12]) For a domain $\Omega \subset \mathbb{R}^{n}$ and a function $u \in W_{l o c}^{1, n}(\Omega)$ satisfying

$$
\int|\nabla u|^{n-2} \nabla u \nabla \eta \geq 0 \text { for each } \eta \in C_{0}^{\infty}(\Omega) \text { and } \eta \geq 0
$$

is called a weak supersolution to $n$-Laplace equation in $\Omega$. A function $u$ is called a weak subsolution if $-u$ is a weak supersolution.

In the mean time, the following definition for $n$-superharmonic functions is often used in nonlinear potential theory. To avoid confusions, we quote the following definition for $n$-superharmonic functions.

Definition 2.3 ([48, Section 2]) For a domain $\Omega \subset \mathbb{R}^{n}$ and a lower semi-continuous function

$$
u: \Omega \rightarrow(-\infty,+\infty]
$$

is said to be $n$-superharmonic in $\Omega$ if $u$ is not identically infinite in each connected component of $\Omega$, and if for all bounded open sets $D \subset \bar{D} \subset \Omega$ and all $h \in C(\bar{D})$ that is $n$-harmonic in $D, h \leq u$ in $\partial D$ implies that $h \leq u$ in $D$. A function $u$ is said to be $n$-subharmonic in $\Omega$ if $-u$ is $n$-superharmonic in $\Omega$.

Fortunately, the relations between these two definitions has been clarified very well in $[38,48]$. For instance, we have

\section{Lemma 2.1 ([48, Proposition 2.7])}

- If $u$ is a weak supersolution to $n$-Laplace equation in $\Omega \subset \mathbb{R}^{n}$, then there is an $n$ superharmonic function $v$ such that $v=u$ a.e. in $\Omega$;

- If $u$ is $n$-superharmonic in $\Omega$ and $u \in W_{l o c}^{1, n}(\Omega)$, then $u$ is a weak supersolution to $n$-Laplace equation;

- If $u$ is $n$-superharmonic and locally bounded, then $u \in W_{l o c}^{1, n}(\Omega)$ and is a weak $n$ supersolution to $n$-Laplace equation.

Clearly, when functions are $C^{2}$ or better, these two definitions agree, we will simply refer them $n$-superharmonic with no confusion. For $n$-superharmonic functions, one still has integrability of the gradient as shown in [51, Theorem 5.15].

Lemma 2.2 ([51, Theorem 5.15]) Suppose that $u$ is an n-superharmonic function in $\Omega$. Let $D \subset \subset \bar{D} \subset \Omega$ be a bounded subdomain and $0<q<n$. Then there is a constant $C>0$ such that

$$
\int_{D}|\nabla u|^{q} d x \leq C .
$$

Therefore, if $u$ is $n$-superharmonic or weak supersolution to the $n$-Laplace equation, then $\mu=-\Delta_{n} u$ may be considered to be a nonnegative Radon measure on $\Omega$ (cf. [51] and [47, Theorem 2.1]). And, by a simple approximation argument,

$$
\int|\nabla u|^{n-2} \nabla u \cdot \nabla \phi=\int \phi d \mu
$$


for any testing function $\phi \in W_{0}^{1, n}(D)$, if $u \in W^{1, n}(D)$ and $D \subset \Omega$. It is also helpful to mention the following weak comparison principle from Theorem 2.15 and the remark right after the proof in [51].

Theorem 2.1 ([51, Theorem 2.15]) Suppose that $u$ is a weak supersolution to the $n$-Laplace equation and $v$ is an $n$-harmonic function in a bounded domain $\Omega \subset \mathbb{R}^{n}$. Iffor every $\zeta \in \partial \Omega$

$$
\limsup _{x \rightarrow \zeta} v(x) \leq \liminf _{x \rightarrow \zeta} u(x)
$$

with the possibilities $\infty \leq \infty$ and $-\infty \leq-\infty$ excluded, then $u \geq v$ almost everywhere in $\Omega$.

For more basic properties of $n$-superharmonic functions, we refer readers to $[38,39,46$, 48,51].

\subsection{The story in $\mathbf{2}$ dimensions}

Thanks to the seminal paper [43] of Huber in 1957 (see also [10,24,31,41]), the connection between geometric properties of surfaces and potential theory based on Gauss curvature equations has been a major part of the theory of surfaces. The Gauss curvature equation in an isothermal coordinates on a surface is

$$
-\Delta u=K e^{2 u}
$$

where $K$ is the Gauss curvature of the surface metric $e^{2 u}|d x|^{2}$. Let us focus on one thread of developments on this subject: local behavior of superharmonic functions near an isolated singular point, or equivalently, asymptotic behavior at infinity of superharmonic functions on the entire plane.

A function that is subharmonic on the entire plane is representable as a function of potential type

$$
v(z)=\int_{\mathbb{C}} \log \left|1-\frac{z}{\xi}\right| d \mu(\xi)
$$

for $z, \xi \in \mathbb{C}$ the complex plane, where $\mu$ is a positive mass distribution and vanishes in a neighborhood of the origin for our purposes. To describe the asymptotic behavior of the function $v$ at infinity one aims to understand the limit

$$
\lim _{z \rightarrow \infty} \frac{v(z)}{\log |z|}
$$

In this regard, notions of thinness play the natural and important role. Notions of thinness at a point was considered by Brelot in [13] in 1940, where a subset $E$ in $\mathbb{C}$ is said to be thin at a point $z_{0}$ if either $z_{0} \notin \bar{E}$ or there exists a subharmonic function $v$ in a neighborhood of $z_{0}$ such that

$$
\limsup _{z \in E \text { and }} v(z)<v\left(z_{0}\right),
$$

which we will refer it as thinness by Cartan property (cf. [6]). This notion of thinness at a point is for potential functions with no point charge at the point. 
In $[6,(1.8)]$, a subset $E$ of $\mathbb{C}$ is said to be thin at infinity if either it is bounded or there exists a function that is subharmonic on the entire complex plane $\mathbb{C}$ such that

$$
\limsup _{z \in E \text { and } z \rightarrow \infty} \frac{v(z)}{\log |z|}<\limsup _{z \rightarrow \infty} \frac{v(z)}{\log |z|} .
$$

At the end of [6], there was a discussion about the correlation of these two notions of thinness. For a function $v$ of potential type, one may take an inversion and consider the subharmonic function

$$
u(z)=v\left(\frac{1}{z}\right)+M \log \frac{1}{|z|}
$$

on the punctured plane with no charge at the origin, where $M$ is the total mass of the potential function $v$. Then (2.6) is equivalent to

$$
\limsup _{z \in \tilde{E} \text { and } z \rightarrow 0} \frac{u(z)}{\log \frac{1}{|z|}}<\limsup _{z \rightarrow 0} \frac{u(z)}{\log \frac{1}{|z|}}=0,
$$

where $\tilde{E}=\left\{\frac{1}{z}: z \in E\right\}$. This is to say that the thinness defined in [6, (1.8)] is the one for potential functions with point charge. It was then pointed out in [6] that $E$ is thin at infinity by (2.6) if and only if $\tilde{E}$ is thin at the origin by (2.5) thanks to [14, Theorem 2]. These two types of thinness can be shown to be no longer equivalent for a nonlinear potential theory, which will be included in our future work.

In the geometric viewpoint, more interestingly, an equivalent criterion for a set to be thin at infinity using log-capacity was established as a Wiener type criterion in [6].

Theorem 2.2 ([6, Theorem 1.3]) Let $E$ be a Borel subset set in the plane and $\gamma_{n}$ be the logarithmic capacity of the part of $E$ lying in the annulus $\left\{z \in \mathbb{C}: r^{n}<|z| \leq r^{n+1}\right\}$ for a fixed number $r>1$ and $n=1,2,3, \cdots$. Then $E$ is thin at infinity if and only if $\gamma_{n} \rightarrow 0$ as $n \rightarrow \infty$ and

$$
\sum_{n=1}^{\infty} \frac{n}{\log \frac{1}{\gamma_{n}}}<\infty
$$

In summary, based on works in $[6,13,14]$ (see also $[5,36,37,40,42]$ ), one knows that, for a function $v$ of potential type, there is a set $E$ that is thin at infinity and

$$
\lim _{z \notin E \text { and } z \rightarrow \infty} \frac{v(z)}{\log |z|}=\limsup _{z \rightarrow \infty} \frac{v(z)}{\log |z|} .
$$

Naturally, one asks what is the condition for a function of potential type to have a clean asymptotic behavior (2.9) with no exceptional thin set $E$ ? This question was solved recently analytically in [63, Theorem 2.1] in 2006 and geometrically in [11, Lemma 4.2] in 2016. Namely,

Theorem 2.3 ([11, Lemma 4.2]) Suppose that $\left(\mathbb{C}, e^{2 u}|d z|^{2}\right)$ is complete with nonnegative and bounded Gauss curvature. Then

$$
u(z)=m \log \frac{1}{|z|}+o(\log |z|) \text { as }|z| \rightarrow \infty
$$

for $m \in[0,1]$. 
It is known that $m=\frac{1}{2 \pi} \int_{\mathbb{C}} K e^{2 u} d z$ and $m \in[0,1]$ due to [24,43], where $m=0$ implies $u$ is a constant. The proof of the above result in [11] relies on two important ingredients that are deep results in geometric analysis and partial differential equation. One is the noncollapsing result of Croke-Karcher [26, Theorem A] in 1988 for complete surfaces with nonnegative Gauss curvature; the other is asymptotic estimates for nonnegative solutions to Gauss curvature type equations of Taliaferro in [63, Theorem 2.1] (see also his previous work [61,62]). One of the key analytic ingredients in [61-63] is the Brezis-Merle inequality of Moser-Trudinger type

$$
\int_{\Omega} e^{\frac{(4 \pi-\delta)|u(x)|}{\|\Delta u\|} L^{1}(\Omega)} d x \leq(\operatorname{diam}(\Omega))^{2} \frac{4 \pi^{2}}{\delta}
$$

for $\left.u\right|_{\partial \Omega}=0$ and $\delta \in(0,4 \pi)$, established in [15, Theorem 1] (cf. [44]).

Taliaferro's estimates in [61-63] are the major work in the theory of local behavior of a class of subharmonic functions near an isolated singular point. And [11, Lemma 4.2] turns out to be essential to the proof of [11, Main Theorem] in 2 dimensions that a complete, nonnegatively curved, immersed surface in hyperbolic 3-space is necessarily properly embedded, except coverings of equidistant surfaces, which was conjectured by Epstein and AlexanderCurrier in [3,4,28-30] around 1990.

\subsection{Isolated singularity for nonnegative $n$-superharmonic functions}

There have been significant developments of the study on local and global behaviors for solutions to (degenerate) quasilinear elliptic equations that include the study of $n$-Laplace equations, for example, $[9,49,65]$ and references therein. The following result on the isolated singularities of nonnegative $n$-superharmonic functions are usually of importance.

Theorem 2.4 ([9, Proposition 1.1]) Let $0<r<R$. Suppose that $w$ is a nonnegative $n$ superharmonic function on the punctured ball $B(0, R) \backslash\{0\}$. Assume that $w$ is continuous and $|\nabla w|^{n},-\Delta_{n} w$ are locally integrable in $B(0, R) \backslash\{0\}$. If $\lim _{x \rightarrow 0} w(x)=\infty$, then there are a function $g \in L^{1}(B(0, r))$ and a number $\beta \geq 0$ such that

$$
-\Delta_{n} w=g+\beta \delta_{0}, x \in B(0, r)
$$

in the distributional sense, where $\delta_{0}$ is the Dirac function at the origin. And $|\nabla w| \in$ $L^{p}(B(0, r))$ for $p \in(0, n)$.

Remark 2.1 We remark here that the function $w$ in the above theorem is in fact $n$ superharmonic in the ball $B(0,1)$ as the potential of the nonnegative Radon measure that is induced from $g+\beta \delta$.

The other important contribution in the study of isolated singularity of $n$-harmonic functions is the following result in $[49,50]$, which is based on previous works in $[59,60,64]$.

Theorem 2.5 ([49, Theorem 1.1]) Suppose that $u$ is a nonnegative n-harmonic function on the punctured ball $B\left(0, r_{0}\right) \backslash\{0\}$. Then, there is a number $\gamma$ such that

$$
u(x)-\gamma \log \frac{1}{|x|} \in L_{l o c}^{\infty}\left(B\left(0, r_{0}\right)\right) .
$$

The idea of the proof of this theorem in [49] is particularly helpful to us. In fact, in some sense, what we would like to have is the extension of this theorem to cover $n$-superharmonic functions. Our approach combines that in [49] and the use of the nonlinear potential theory $[2,39,48,51,52]$. 


\subsection{Non-linear potential theory for $n$-Laplace equations}

The nonlinear potential theory itself is a vast and profound subject in Mathematics. We certainly do not intend to give an comprehensive introduction here. Instead we will collect useful facts in a cohesive way. To study $n$-Laplace equations

$$
-\Delta_{n} w=\mu
$$

where $\mu$ is a nonnegative Radon measure representing the mass distribution, there is the nonlinear potential theory developed to replace the principle of superposition (cf. [2,38,39, $48,51,52])$. The fundamental tool is the Wolff potential

$$
W_{1, n}^{\mu}\left(x_{0}, r\right)=\int_{0}^{r} \mu\left(B\left(x_{0}, t\right)\right)^{\frac{1}{n-1}} \frac{d t}{t} .
$$

The Wolff potential plays the same role in the nonlinear potential theory as the Riesz potential plays in the linear one. The foundational estimates in the nonlinear potential for the equation (2.14) are as follows:

Theorem 2.6 ([48, Theorem 1.6]) Suppose that $w$ is a nonnegative $n$-superharmonic function satisfying (2.14) for a nonnegative Radon measure $\mu$ in $B\left(x_{0}, 3 r\right)$. Then

$$
C_{1} W_{1, n}^{\mu}\left(x_{0}, r\right) \leq w\left(x_{0}\right) \leq C_{2} \inf _{B\left(x_{0}, r\right)} w+C_{3} W_{1, n}^{\mu}\left(x_{0}, 2 r\right)
$$

for some dimensional constants $C_{1}, C_{2}, C_{3}>0$.

It is easily seen that the study of $n$-Laplace equations is intimately related to $n$-capacity since solutions to $n$-Laplace equations are critical points for the functional $\int|\nabla u|^{n} d x$. We therefore recall the definition of $n$-capacity from [48, Section 3].

Definition 2.4 For a compact subset $K$ of a domain $\Omega$ in Euclidean space $\mathbb{R}^{n}$, we define

$$
\operatorname{cap}_{n}(K, \Omega)=\inf \int_{\Omega}|\nabla u|^{n} d x
$$

for all $u \in C_{0}^{\infty}(\Omega)$ and $u \geq 1$ on $K$. Then $n$-capacity for arbitrary subset $E$ of $\Omega$ is

$$
\operatorname{cap}_{n}(E, \Omega)=\inf _{\text {open } G \subset \Omega} \sup _{\text {that contains } E \text { compact } K \subset G} \operatorname{cap}_{n}(K, \Omega) .
$$

The $n$-capacity is clearly invariant under conformal transformations, and therefore is also called the conformal capacity (cf. [33], for example). The notions of $n$-thinness in the potential theory are important in the study of $n$-superharmonic functions. Notions of $n$ thinness were first considered in [2], and readers are referred to [2,38,48] for more background and references. One notion of $n$-thinness is defined via Wiener integral given in $[2,48]$, which we will refer to as thinness by Wiener integral. One of the major achievements in [48] is to establish the complete equivalence between the thinness by Wiener integral and the one by Cartan property (2.5) in general dimensions, based on [48, Theorem 1.6] and early works $[2,38]$. But, these notions of thinness at a point are for potential functions with no point charge, which is only known to be the same as the notion of thinness for potential functions with point charge in 2 dimensions ( [14, Theorem 2] and [6]). In higher dimensions, inspired by [6, Theorem 1.3], we will introduce a new notion of thinness using $n$-capacity and study its relation to the Cartan property (2.6) for $n$-subharmonic functions at isolated singular point (cf. Definition 3.1 and Theorem 3.1 in next section). 


\section{$2.5 n$-Laplace equations in differential geometry}

What can we do in higher dimensions following the approach in [43] by Huber? We have seen successful efforts, for example, in $[17,18,22,58,67]$ to explore higher dimensional counterparts of Gauss curvature equations (2.4) such as the scalar curvature equations

$$
-\frac{4(n-1)}{n-2} \Delta u+R u=\bar{R} u^{\frac{n+2}{n-2}}
$$

where $R$ and $\bar{R}$ are scalar curvature of the metrics $g$ and $\bar{g}=u^{\frac{4}{n-2}} g$ respectively in dimensions $n \geq 3$; and the higher order analogue: $Q$-curvature equations,

$$
P_{n} w+Q_{n}=\bar{Q}_{n} e^{2 n w},
$$

where $P_{n}=(-\Delta)^{n}+$ lower order is the so-called Paneitz type operator and $Q_{n}, \bar{Q}_{n}$ are so-called $Q$-curvature of the metrics $g$ and $\bar{g}=e^{2 w} g$ respectively in dimensions $2 n \geq 2$. We have also seen remarkable successes in using fully nonlinear equations of Weyl-Schouten curvature, as replacements of Gauss curvature equations, in [20,21,34,35].

\subsection{1 n-Laplace equations in conformal geometry}

Recall the change of Ricci curvature under conformal change of metrics is

$$
\bar{R}_{i j}=R_{i j}-\Delta \phi g_{i j}+(2-n) \phi_{i j}+(n-2) \phi_{i} \phi_{j}+(2-n)|\nabla \phi|^{2} g_{i j},
$$

where $R_{i j}, \bar{R}_{i j}$ are Ricci curvature tensors for the metrics $g$ and $\bar{g}=e^{2 \phi} g$ respectively in $n$ dimensions. Contracting with $\phi^{i}$ and $\phi^{j}$ on both sides of the above equation, one gets that

$$
\phi^{i} \phi^{j} \bar{R}_{i j}=\phi^{i} \phi^{j} R_{i j}-|\nabla \phi|^{4-n} \operatorname{div}\left(|\nabla \phi|^{n-2} \nabla \phi\right) .
$$

Therefore one arrives at another generalization of Gauss curvature equations in higher dimensions,

$$
-\operatorname{div}\left(|d \phi|^{n-2} \nabla \phi\right)+|\nabla \phi|^{n-2} \operatorname{Ric}\left(\frac{\nabla \phi}{|\nabla \phi|}, \frac{\nabla \phi}{|\nabla \phi|}\right)=|\bar{\nabla} \phi|_{\bar{g}}^{n-2} \bar{R} i c\left(\frac{\bar{\nabla} \phi}{|\bar{\nabla} \phi|_{\bar{g}}}, \frac{\bar{\nabla} \phi}{|\bar{\nabla} \phi|_{\bar{g}}}\right) e^{n \phi} .
$$

In particular, when $g$ is Ricci-flat, we have

$$
-\Delta_{n} \phi=|\bar{\nabla} \phi|_{\bar{g}}^{n-2} \bar{R} i c\left(\frac{\bar{\nabla} \phi}{|\bar{\nabla} \phi|_{\bar{g}}}, \frac{\bar{\nabla} \phi}{|\bar{\nabla} \phi|_{\bar{g}}}\right) e^{n \phi} .
$$

In this paper we want to explore properties of $n$-superharmonic functions and the geometric consequences. Following the approach in [43] by Huber we want to extend the success in 2 dimensions to higher dimensions and complement contemporary developments in conformal geometry and conformally invariant partial differential equations.

\subsubsection{Hypersurfaces in hyperbolic space}

Apparently, the first use of $n$-subharmonic functions in differential geometry was in [12] to overcome the limitation of the use of subharmonic functions in 2 dimensions or sectional curvature assumptions. Inspired by the calculation in $[3,4]$, it was calculated and concluded 
Theorem 2.7 ([12, Theorem 3.1]) The height function in Busemann coordinates for a hypersurface with nonnegative Ricci curvature in hyperbolic space is $n$-subharmonic.

It is perhaps worth to mention, for immersed hypersurfaces $\Sigma^{n} \subset \mathbb{H}^{n+1}$ with appropriate orientation, the following successively stronger pointwise convexity conditions on the principal curvatures $\kappa_{1}, \ldots, \kappa_{n}$ :

$$
\begin{array}{ll}
\kappa_{i}>0 & \text { strict convexity } \\
\kappa_{i}\left(\sum_{l=1}^{n} \kappa_{l}\right)-\kappa_{i}^{2} \geq n-1 & \text { nonnegative Ricci curvature } \\
\kappa_{i} \kappa_{j} \geq 1 & \text { nonnegative sectional curvature for } i \neq j
\end{array}
$$

This observation enables the authors in [12] to improve the end structure theorem of $[3,4]$ as follows:

Theorem 2.8 ([12, Main Theorem $]$ ) For $n \geq 3$, suppose that $\Sigma$ is a complete and noncompact hypersurface with nonnegative Ricci curvature properly embedded in hyperbolic space $\mathbb{H}^{n+1}$. Then $\partial_{\infty} \Sigma$ consists of at most two points. The case that $\partial_{\infty} \Sigma$ consists of two points is a rigidity condition that forces $\Sigma$ to be an equidistant hypersurface about a geodesic line.

In this paper we will use the properties of $n$-superharmonic functions to derive asymptotic behaviors for hypersurfaces in hyperbolic space with nonnegative Ricci and improve the asymptotic results in $[3,4]$.

\section{Higher dimensional Arsove-Huber's theorem}

In this section our goal is to extend Theorem 2.2 and Eq. (2.9) (cf. [6, Theorem 1.3]) to general dimensions. First we define a notion of thinness by capacity inspired by that in 2 dimensions in [6, Theorem 1.3] for potential functions with point charge.

For $x_{0} \in \mathbb{R}^{n}$, we set

$$
\begin{aligned}
& \omega\left(x_{0}, i\right)=\left\{x \in \mathbb{R}^{n}: 2^{-i-1} \leq\left|x-x_{0}\right| \leq 2^{-i}\right\} \text { and } \\
& \Omega\left(x_{0}, i\right)=\left\{x \in \mathbb{R}^{n}: 2^{-i-2}<\left|x-x_{0}\right|<2^{-i+1}\right\} .
\end{aligned}
$$

And we set

$$
\begin{aligned}
& \omega(\infty, i)=\left\{x \in \mathbb{R}^{n}: 2^{i} \leq|x| \leq 2^{i+1}\right\} \text { and } \\
& \Omega(\infty, i)=\left\{x \in \mathbb{R}^{n}: 2^{i-1}<|x|<2^{i+2}\right\} .
\end{aligned}
$$

Definition 3.1 Let $E \subset \mathbb{R}^{n}$ and $x_{0} \in \mathbb{R}^{n}$. We say $E$ is $n$-thin at $x_{0}$, if

$$
\sum_{i=1}^{\infty} i^{n-1} \operatorname{cap}_{n}\left(E \cap \omega\left(x_{0}, i\right), \Omega\left(x_{0}, i\right)\right)<+\infty .
$$

Clearly $E$ is trivially $n$-thin if $x_{0} \notin \bar{E}$. Similarly, we say $E$ is $n$-thin at $\infty$, if

$$
\sum_{i=1}^{\infty} i^{n-1} \operatorname{cap}_{n}(E \cap \omega(\infty, i), \Omega(\infty, i))<+\infty .
$$

Again, $E$ is trivially $n$-thin at $\infty$ if $E$ is bounded. 
Clearly the inversion $\frac{x}{|x|^{2}}$ of $\mathbb{R}^{n}$ takes a subset $E \subset \mathbb{R}^{n}$ that is $n$-thin at infinity to a subset $\tilde{E}$ that is $n$-thin at the origin. For the convenience of readers, we repeat our first main theorem in the introduction as follows:

Theorem 3.1 Let $w$ be a nonnegative lower semi-continuous function that is $n$-superharmonic in $B(0,2) \subset \mathbb{R}^{n}$ and

$$
-\Delta_{n} w=\mu \geq 0
$$

for a Radon measure $\mu \geq 0$. Then there is a set $E \subset \mathbb{R}^{n}$, which is $n$-thin at the origin, such that

$$
\lim _{x \notin E \text { and }|x| \rightarrow 0} \frac{w(x)}{\log \frac{1}{|x|}}=\liminf _{|x| \rightarrow 0} \frac{w(x)}{\log \frac{1}{|x|}}=m \geq 0
$$

and

$$
w(x) \geq m \log \frac{1}{|x|}-C \text { for } x \in B(0,1) \backslash\{0\} \text { and some } C .
$$

Moreover, if $w \in C^{2}(B(0,2) \backslash\{0\})$ and $\left(B(0,2) \backslash\{0\}, e^{2 w}|d x|^{2}\right)$ is complete at the origin, then $m \geq 1$.

To study the local behavior for a nonnegative $n$-superharmonic function $w$ on the punctured ball, we follow the idea from [49] to consider the blow-down

$$
w_{r}(\xi)=\frac{w(r \xi)}{\log \frac{1}{r}} \text { for } \xi \in B\left(0, \frac{2}{r}\right) \backslash\{0\} \text { as } r \rightarrow 0 .
$$

\subsection{The first step in the proof of Theorem 3.1}

The first observation we need is that the quotient $\frac{w(x)}{\log \frac{1}{|x|}}$ is uniformly bounded away from a "small" subset. Therefore the following proposition is the first key step to prove Theorem 3.1.

Proposition 3.1 Assume the same assumptions as in Theorem 3.1. Then there is a set $\hat{E}$, which is $n$-thin at the origin, and a constant $\hat{C}$ such that

$$
0 \leq \frac{w(x)}{\log \frac{1}{|x|}} \leq \hat{C}
$$

for all $x \in(B(0,1) \backslash\{0\}) \backslash \hat{E}$.

The proof of Proposition 3.1 starts with the following simple fact observed in [48, Lemma 3.9].

Lemma 3.1 ([48, Lemma 3.9]) Suppose that $u$ is an n-superharmonic function satisfying $\min \{u, \lambda\} \in W_{0}^{1, n}(\Omega)$ for $\forall \lambda>0$ and

$$
-\Delta_{n} u=\mu
$$

for a nonnegative Radon measure $\mu$. Then, for $\lambda>0$,

$$
\lambda^{n-1} \operatorname{cap}_{n}(\{x \in \Omega: u(x)>\lambda\}, \Omega) \leq \mu(\Omega) .
$$


The proof of Lemma 3.1 is to use $\frac{\min \{u, \lambda\}}{\lambda}$ as a test function and get

$$
\int_{\Omega}|\nabla \min \{u, \lambda\}|^{n} \leq \frac{\mu(\Omega)}{\lambda^{n-1}},
$$

which is easily seen to imply the above $n$-capacity estimate (3.3).

The next fact we need to prove Proposition 3.1 is the following basic existence result (cf. [47, Theorem 2.4]).

Lemma 3.2 ([47, Theorem 2.4]) For a bounded domain $\Omega \subset \mathbb{R}^{n}$ and a nonnegative finite Radon measure $\mu$, there always exists a solution $u(x) \geq 0$ to the equation

$$
-\Delta_{n} u=\mu \text { in } \Omega
$$

and $\min \{u, \lambda\} \in W_{0}^{1, n}(\Omega)$ for any $\lambda>0$.

To make use of the fundamental estimates (2.16) in Theorem 2.6 (cf. [48, Theorem 1.6]), we also need the following estimates on the infimum.

Lemma 3.3 Suppose that $w \geq 0$ satisfies

$$
-\Delta_{n} w=\mu \geq 0 .
$$

Then there is a constant $C>0$ such that

$$
\inf _{B\left(x_{0}, \frac{\left|x_{0}\right|}{2}\right)} w(x) \leq C\left(n,\|w\|_{L^{n}(B(0,1))}\right) \log \frac{1}{\left|x_{0}\right|}
$$

for each $x_{0} \in B\left(0, \frac{1}{2}\right) \backslash\{0\}$.

Proof We will rely on some estimates from [27, Section 7] to derive this lemma. Readers are referred to [27] for definitions and notations. Particularly, in the light of [27, Lemma 14 and 15], we know that

$$
\|w\|_{B M O\left(B\left(0, \frac{1}{4}\right), B\left(0, \frac{1}{2}\right)\right)} \leq C_{1}(n)\left(1+\|w\|_{L^{n}\left(B\left(0, \frac{1}{2}\right)\right)}\right) .
$$

Meanwhile, from [51, Theorem 5.11], for instance, we know $\|w\|_{L^{n}\left(B\left(0, \frac{1}{2}\right)\right.}$ is finite. Therefore $\|w\|_{B M O\left(B\left(0, \frac{1}{4}\right), B\left(0, \frac{1}{2}\right)\right)}$ is finite. Here we remark that in [27, Section 7], the assumption that the right hand side in $L^{1}$ can be generalized to a nonnegative Radon measure easily. And the assumption that $w \in W^{1, n}$ is not essential, because, if not, we can replace $w$ by $\min \{u, k\}$, which belongs to $W^{1, n}$, and use Fatou's lemma to prove (3.5) for $w$.

Suppose otherwise that (3.4) were not true. Then we have a sequence

$$
\left\{x_{i} \in B\left(0, \frac{1}{4}\right): x_{i} \rightarrow 0\right\}
$$

such that

$$
\inf _{B\left(x_{i}, \frac{1}{2}\left|x_{i}\right|\right)} w(x)>C\left(n,\|w\|_{L^{n}(B(0,1))}\right) \log \frac{1}{\left|x_{i}\right|},
$$

for some $C\left(n,\|w\|_{L^{n}(B(0,1))}\right)$ to be fixed. We let

$$
\mu_{i}=\mu\left(\left\{x \in B\left(0, \frac{1}{4}\right): w(x)-\bar{w}>\frac{C\left(n,\|w\|_{L^{n}(B(0,1))}\right)}{2} \log \frac{1}{\left|x_{i}\right|}\right\}\right),
$$


where the finite number $\bar{w}$ is the average of $w$ on $B\left(0, \frac{1}{4}\right)$. Clearly, at least for $i$ large,

$$
\mu_{i} \geq C(n)\left|x_{i}\right|^{n}
$$

because of (3.6). On the other hand, from [45, Lemma 1], we know that there are $B(n)$ and $b(n)$, such that

$$
\mu_{i} \leq B(n) \mu\left(B\left(0, \frac{1}{2}\right)\right)\left|x_{i}\right|^{\frac{b(n) C\left(n,\|w\|_{L^{n}(B(0,1))}\right.}{2\|w\|}}{ }_{B M O\left(B\left(0, \frac{1}{4}\right), B\left(0, \frac{1}{2}\right)\right)} .
$$

Now we choose

$$
C\left(n,\|w\|_{L^{n}(B(0,1))}\right)=2(n+1) b(n)^{-1} C_{1}(n)\left(1+\|w\|_{L^{n}(B(0,1))}\right)
$$

and get $\mu_{i} \leq B(n) \mu\left(B\left(0, \frac{1}{2}\right)\right)\left|x_{i}\right|^{n+1}$, which is a contradiction with (3.7). Thus the proof is completed.

For convenience and simplicity, we use

$$
\begin{aligned}
& \omega_{i}=\omega(0, i)=\left\{x \in \mathbb{R}^{n}: 2^{-i-1} \leq|x| \leq 2^{-i}\right\}=2^{-i} \omega(0,0) \\
& \Omega_{i}=\Omega(0, i)=\left\{x \in \mathbb{R}^{n}: 2^{-i-2}<|x|<2^{-i+1}\right\}=2^{-i} \Omega(0,0) .
\end{aligned}
$$

Then

$$
\frac{i}{2} \log 2 \leq(i-1) \log 2 \leq \log \frac{1}{|x|} \leq(i+2) \log 2 \leq 2 i \log 2 \text { for all } x \in \Omega_{i} .
$$

Now we are ready to start the proof of Proposition 3.1.

The proof of Proposition 3.1 It is obvious that $\frac{w(y)}{\log \frac{1}{|y|}} \geq 0$. We are going to prove that outside some set $\hat{E}$, which is $n$-thin at the origin, the quotient $\frac{w(y)}{\log \frac{1}{|y|}}$ has upper bound.

We cover $\omega_{0}$ with finite number of balls $\left\{B_{1}^{0}, \ldots, B_{m}^{0}\right\}$, where the center of $B_{j}^{0}$ lies in $\omega_{0}$, the concentric ball $4 B_{j}^{0} \subset \Omega_{0}$ for $j=1, \ldots, m$, and $m$ depends only on the dimension $n$. For $i \geq 0$, we denote $B_{j}^{i}=2^{-i} B_{j}^{0}$. It's obvious that $\left\{B_{j}^{i}: j=1, \ldots, m\right\}$ cover $\omega_{i}$ and each $4 B_{j}^{i}$ lie in $\Omega_{i}$. We let $r_{i j}$ be the radius of $B_{j}^{i}$. Clearly $r_{i j}=2^{-i} r_{0 j}$.

For any $y \in B_{j}^{i}$, from [48, Theorem 1.6] and Lemma 3.3, we have

$$
w(y) \leq C_{2}(n) \inf _{B\left(y, \frac{|y|}{8}\right)} w+C_{3}(n) W_{1, n}^{\mu}\left(y, \frac{|y|}{4}\right)
$$

Since $|y| \sim r_{i j} \sim 2^{-i}$ and

$$
\left|W_{1, n}^{\mu}\left(y, \frac{|y|}{4}\right)-W_{1, n}^{\mu}\left(y, \frac{1}{3} r_{i j}\right)\right|=\left|\int_{\frac{1}{3} r_{i j}}^{\frac{|y|}{4}} \mu(B(y, t))^{\frac{1}{n-1}} \frac{d t}{t}\right| \leq C,
$$

we arrive at

$$
w(y) \leq C\left(\log \frac{1}{|y|}+W_{1, n}^{\mu}\left(y, \frac{1}{3} r_{i j}\right)+1\right) .
$$


To estimate $W_{1, n}^{\mu}\left(y, \frac{1}{3} r_{i j}\right)$, we use Lemma 3.2 and solve the following

$$
\left\{\begin{array}{l}
-\Delta_{n} w_{i j}(y)=\mu, \text { in } 2 B_{j}^{i}(y) \\
\left.w_{i j}(y)\right|_{\partial\left(2 B_{j}^{i}\right)}=0 .
\end{array}\right.
$$

The advantage is that, from [48, Lemma 3.9], we know that

$$
\operatorname{cap}_{n}\left(\left\{y \in B_{j}^{i}: w_{i j}(y)>\log \frac{1}{|y|}\right\}, 2 B_{j}^{i}\right) \leq \frac{C \mu\left(2 B_{j}^{i}\right)}{i^{n-1}} .
$$

Now, using [48, Theorem 1.6] again, we have

$$
C_{1} W_{1, n}^{\mu}\left(y, \frac{1}{3} r_{i j}\right) \leq w_{i j}(y), \quad \forall y \in B_{j}^{i},
$$

which implies that

$$
\operatorname{cap}_{n}\left(\left\{y \in B_{j}^{i}: W_{1, n}^{\mu}\left(y, \frac{1}{3} r_{i j}\right)>\frac{1}{C_{1}} \log \frac{1}{|y|}\right\}, 2 B_{j}^{i}\right) \leq \frac{C \mu\left(2 B_{j}^{i}\right)}{i^{n-1}} .
$$

Let

$$
\hat{E}_{i j}=\left\{y \in B_{j}^{i}: W_{1, n}^{\mu}\left(y, \frac{1}{3} r_{i j}\right)>\frac{1}{C_{1}} \log \frac{1}{|y|}\right\} \cap \omega_{i}
$$

and

$$
\hat{E}_{i}=\cup_{j} \hat{E}_{i j} \quad \hat{E}=\cup_{i} \hat{E}_{i}
$$

Then we have

$$
\operatorname{cap}_{n}\left(\hat{E}_{i j}, \Omega_{i}\right) \leq \operatorname{cap}_{n}\left(\hat{E}_{i j}, 2 B_{j}^{i}\right) \leq \frac{C \mu\left(2 B_{j}^{i}\right)}{i^{n-1}} .
$$

Hence from Theorem 2.2 (vi) of [39]

$$
\operatorname{cap}_{n}\left(\hat{E} \cap \omega_{i}, \Omega_{i}\right) \leq \sum_{j} \operatorname{cap}_{n}\left(\hat{E}_{i j}, \Omega_{i}\right) \leq \frac{C \mu\left(\Omega_{i}\right)}{i^{n-1}} .
$$

Therefore

$$
\sum_{i} i^{n-1} \operatorname{cap}_{n}\left(\hat{E} \cap \omega_{i}, \Omega_{i}\right) \leq C \mu\left(B_{1}(0) \backslash\{0\}\right)<+\infty .
$$

Thus, from (3.8), there is a constant $\hat{C}>0$ such that, outside $\hat{E}$, which is $n$-thin according to Definition 3.1, (3.2) holds. The proof is completed.

\subsection{The second step in the proof of Theorem 3.1}

The second key step in the proof of Theorem 3.1, for the sake of the blow-down argument as the one used in [49], is to modify the function $\frac{w(r \xi)}{\log \frac{1}{r}}$ to accommodate the lack of boundedness. We use the trick from [27] and consider the cut-off function

$$
a_{\alpha}(s)=\left\{\begin{array}{cl}
s & \text { when } 0 \leq s \leq \alpha \\
\alpha+\int_{\alpha}^{s}\left(\frac{\alpha}{t}\right)^{\frac{n}{n-1}} d t & \text { when } s>\alpha,
\end{array}\right.
$$


where $\alpha$ is to be fixed as $\hat{C}+1$ throughout this paper, where $\hat{C}$ is the one in (3.2). One may calculate that

$$
\begin{aligned}
a_{\alpha}(s) t \leq n \alpha & \left\{\begin{array}{c}
1 \quad \text { when } 0 \leq s \leq \alpha \\
\left(\frac{\alpha}{s}\right)^{\frac{n}{n-1}} \text { when } s>\alpha,
\end{array}\right. \\
a_{\alpha}^{\prime}(s) & \begin{array}{r}
0 \quad \text { when } 0 \leq s \leq \alpha \\
-\frac{n}{n-1}\left(\frac{\alpha}{s}\right)^{\frac{n}{n-1}} s^{-1} \quad \text { when } s>\alpha,
\end{array} \\
a_{\alpha}^{\prime \prime}(s) & =\left\{\begin{array}{c}
-\Delta_{n} u \quad \text { when } 0 \leq u \leq \alpha \\
-\left(\frac{\alpha}{u}\right)^{n} \Delta_{n} u+n\left(\frac{\alpha}{u}\right)^{n} u^{-1}|\nabla u|^{n}
\end{array} \text { when } u>\alpha .\right.
\end{aligned}
$$

Now we are to carry out the blow-down argument as in [49]. For each $r>0$ and small, we consider the modified blow-down

$$
\hat{w}_{r}(\xi)=a_{\alpha}\left(w_{r}(\xi)\right)=a_{\alpha}\left(\frac{w(r \xi)}{\log \frac{1}{r}}\right) .
$$

Clearly, we have

$$
0 \leq \hat{w}_{r}(\xi) \leq n \alpha=n(\hat{C}+1)
$$

for

$$
\xi \in A_{0, \frac{1}{r}}=\left\{\xi \in \mathbb{R}^{n}:|\xi| \in\left(0, \frac{1}{r}\right)\right\}
$$

and

$$
-\Delta_{n}^{\xi} \hat{w}_{r}(\xi)=\left\{\begin{array}{l}
-\frac{r^{n}}{\left(\log \frac{1}{r}\right)^{n-1}} \Delta_{n}^{x} w(r \xi) \quad \text { for } 0 \leq w_{r}(\xi) \leq \alpha \\
\frac{r^{n}}{\left(\log \frac{1}{r}\right)^{n-1}}\left(\frac{\alpha}{w_{r}(\xi)}\right)^{n}\left(-\Delta_{n}^{x} w(r \xi)+n \frac{1}{w(r \xi)}\left|\nabla^{x} w\right|^{n}(r \xi)\right) \text { for } w_{r}(\xi)>\alpha
\end{array}\right.
$$

for $\xi \in A_{0, \frac{1}{r}}$. To summarize, we state the following lemma to use the above calculations.

Lemma 3.4 Assume the same assumptions as in Theorem 3.1. Then he modified blow-down $\hat{w}_{r}(\xi)$ is a nonnegative and bounded $n$-superharmonic function satisfying

$$
-\Delta_{n}^{\xi} \hat{w}_{r}=\hat{\mu}_{r} \geq 0 \text { in } A_{0, \frac{1}{r}}
$$

for a Radon measure $\hat{\mu}_{r}$ in $A_{0, \frac{1}{r}}$ and $\hat{w}_{r}(\xi) \leq n \hat{C}+n$ for all $x \in A_{0, \frac{1}{r}}$. More importantly, for any fixed $R>1$,

$$
\int_{A_{\frac{1}{R}, R}} d \hat{\mu}_{r}(\xi) \leq\left(\frac{1}{\log \frac{1}{r}}\right)^{n-1} \int_{A_{\frac{r}{R}, r R}} d \mu+n \alpha^{n-1} \int_{A_{\frac{r}{R}, r R} \cap \hat{E}} \frac{|\nabla w|^{n}}{w^{n}} d x,
$$

where $\hat{E}$ is the subset given in Proposition 3.1, which is n-thin at the origin. 
By Lemma 3.4 we want to show that, at least for sequences $r_{k} \rightarrow 0, \hat{w}_{r_{k}}(\xi)$ converges to a bounded $n$-harmonic function on the entire space $\mathbb{R}^{n}$ except possibly the origin, which can only be a constant due to [55] because the origin and the infinity are removable singularities by [59]. To be more precise, we need the following convergence lemma.

Lemma 3.5 Suppose that $\left\{u_{i}\right\}$ is a sequence of $n$-superharmonic functions in a bounded domain $\Omega \subset \mathbb{R}^{n}$ and

$$
-\Delta_{n} u_{i}=\mu_{i} \text { in } \cdot
$$

where $\mu_{i}$ is a sequence of Radon measures. Assume that

$$
0 \leq u_{i} \leq M \text { and } \mu_{i} \rightarrow 0 \text { in the sense of distribution } .
$$

Then, for each bounded subdomain $D \subset \bar{D} \subset \Omega$, there is a constant $C>0$ such that

$$
\int_{D}\left|\nabla u_{i}\right|^{n} d x \leq C
$$

for all $i$ and there is $u \in W^{1, n}(D)$ such that

$$
u_{i} \rightarrow u \text { in } W^{1, n}(D) \text { and }-\Delta_{n} u=0 \text { in } D \text { in distributional sense, }
$$

taking a subsequence if necessary.

Proof For the convenience of readers, we present proof here. First we prove

$$
\int_{D} \frac{\left|\nabla u_{i}\right|^{n}}{\left(u_{i}+1\right)^{2}} d x \leq C \int_{\Omega \backslash D}\left(u_{i}+1\right)^{n-2} d x .
$$

Similar to the argument in [51, Theorem 5.15], based on Lemma 2.1 (cf. [38] and [37, Proposition 2.7]), we simply use the test functions $\zeta^{n}\left(u_{i}+1\right)^{-1}$, where

$$
\zeta \in C_{0}^{\infty}(\Omega), \zeta \equiv 1 \text { on } D, 0 \leq \zeta \leq 1,|\nabla \zeta| \leq \frac{C}{\operatorname{dist}(D, \partial \Omega)} .
$$

Then, from

$$
\int_{\Omega}-\left(\Delta_{n} u_{i}\right) \zeta^{n}\left(u_{i}+1\right)^{-1} \geq 0
$$

we get

$$
\int_{\Omega}\left|\nabla u_{i}\right|^{n} \zeta^{n}\left(u_{i}+1\right)^{-2} \leq n^{n} \int_{\Omega}\left(u_{i}+1\right)^{n-2}|\nabla \zeta|^{n} .
$$

This obviously implies (3.21). Next, to prove (3.20) by (3.21), we derive

$$
\begin{aligned}
\int_{D}\left|\nabla u_{i}\right|^{n} & \leq\left(\sup \left|u_{i}\right|+1\right)^{2} \int_{\Omega}\left|\nabla\left(u_{i}+1\right)\right|^{n} \zeta^{n}\left(u_{i}+1\right)^{-2} \\
& \leq n^{n}\left(\sup \left|u_{i}\right|+1\right)^{2}\left(\sup \left|u_{i}\right|+1\right)^{n-2} \int_{\Omega}|\nabla \zeta|^{n} \\
& \leq C(n, \Omega, D, M) .
\end{aligned}
$$


Hence there is $u \in W^{1, n}(D)$ such that $u_{i} \rightarrow u$ in $W^{1, n}(D)$, at least for a subsequence. In light of

$$
\int_{D}\left|\nabla u_{i}\right|^{n-2} \nabla u_{i} \nabla \phi=\int_{D} \phi d \mu_{i} \rightarrow 0
$$

as $i \rightarrow \infty$ for any $\phi \in C_{0}^{\infty}(D)$, it suffices to prove that

$$
\int_{D}\left|\nabla u_{i}\right|^{n-2} \nabla u_{i} \nabla \phi \rightarrow \int_{D}|\nabla u|^{n-2} \nabla u \nabla \phi
$$

as $i \rightarrow \infty$. Thanks to [66, Theorem 1.1], we know that $u_{i} \rightarrow u$ strongly in $W^{1, p}(D)$ for all $1 \leq p<n$, which implies (3.22). Note that [66, Theorem 1.1] imposed the condition that $-\Delta_{n} u \in L^{1}(\Omega)$. However, if one checks his argument carefully, the only place where this is used is when dealing with (2.7), Page 385. If we replace $f_{k}$ with nonnegative Radon measure $\mu_{k}$ with $\mu_{k}(\Omega) \rightarrow 0$, we can also prove that

$$
\left|\int_{\Omega} w_{k}^{\lambda} d \mu_{k}\right| \leq \lambda\left|\mu_{k}(\Omega)\right| \rightarrow 0 .
$$

Thus the lemma is proved.

Remark 3.1 ([39, Theorem 3.57] [51, Theorem 5.15]) Let $u>1$ be an $n$-superharmonic function in $\Omega$, which is not necessarily bounded from above. From the proof of [51, Theorem 5.15] (please see above), one actually gets

$$
\int \zeta^{n}|\nabla u|^{n} u^{-1-\alpha} d x \leq C(n, \alpha) \int u^{n-1-\alpha}|\nabla \zeta|^{n} d x
$$

for any $\alpha \in(0, n-1]$ and any cut-off function as in the above proof. The right hand side of (3.23) is finite by [51, Theorem 5.11]. This remark is useful to handle the second term on the right side of (3.19).

\subsection{The third step in the proof of Theorem 3.1}

The third key step in the proof of Theorem 3.1 is to show the uniqueness of possible limits of all blow-down sequences. We continue to use the approach used as in [49]. One of the key tools is the following weak comparison principle as a consequence of [64, Lemma 3.1] (please also see [49, Corollary 1.1] and the comment in [50]).

Lemma 3.6 ([64, Lemma 3.1] [49, Corollary 1.1]) Assume $\Omega$ is a connected open subset of $\mathbb{R}^{n} \backslash\{0\}$ and $u$ is $n$-superharmonic in $\Omega$. Then

$$
\inf _{\partial \Omega} \frac{u(x)}{\log \frac{1}{|x|}} \leq \inf _{\Omega} \frac{u(x)}{\log \frac{1}{|x|}} .
$$

For any blow-down sequence $\hat{w}_{r_{i}}(\xi)$ with $r_{i} \rightarrow 0$, there is $\xi_{r_{i}}$ with $\left|\xi_{r_{i}}\right|=1$ and

$$
\hat{w}_{r_{i}}\left(\xi_{r_{i}}\right)=w_{r_{i}}\left(\xi_{r_{i}}\right)=\frac{w\left(r_{i} \xi_{r_{i}}\right)}{\log \frac{1}{r_{i}}}=\min _{|x|=r_{i}} \frac{w(x)}{\log \frac{1}{|x|}} \rightarrow \liminf _{x \rightarrow 0} \frac{w(x)}{\log \frac{1}{|x|}} \leq \hat{C} .
$$


Lemma 3.6 implies that the quotient $\min _{|x|=r} \frac{w(x)}{\log \frac{1}{|x|}}$ is non-increasing as $r \rightarrow 0$, since the infimum is always achieved at the inner sphere of the annulus $B\left(0, r_{0}\right) \backslash B(0, s)$ for $r_{0}<1$ fixed while $s$ arbitrarily small. Notice that we may assume

$$
\lim _{|x| \rightarrow 1^{-}} \frac{w(x)}{\log \frac{1}{|x|}}=\infty
$$

if necessary. Because, when proving Theorem 3.1 one may deal with $w+\epsilon$ for arbitrarily small $\epsilon$ instead. We will present the proof of the uniqueness of all blow-down limits, which is based on Lemma 3.6, in the proof of Theorem 3.1 in next section.

\subsection{The last step of the proof of Theorem 3.1}

With all the preparation we finally are ready to prove Theorem 3.1. At this point, we have cleared almost everything except that the convergences of each blow-down sequence $\hat{w}_{r_{k}}$ to a constant is weaker than the pointwise one. This in principle is caused by the fact that the density function is just a Radon measure $\mu$. Our main goal here, after presenting a proof of the uniqueness of the sequential blow-down limits, is to extract a possible bad set $E$, which is again $n$-thin so that outside $E$ the limit of the quotient $\frac{w(x)}{\log \frac{1}{|x|}}$ is $\lim _{\operatorname{linf}} \rightarrow 0 \frac{w(x)}{\log \frac{1}{|x|}}$ pointwisely.

The proof of Theorem 3.1 To recap, first, from Proposition 3.1 in Sect. 3.1, we know that, outside the thin set $\hat{E}$,

$$
\frac{w(x)}{\log \frac{1}{|x|}} \leq \hat{C} .
$$

Then, based on the discussion in Sect. 3.2, we consider the modified blow-down functions $\hat{w}_{r}(\xi)$ by (3.15) for $\alpha=1+\hat{C}$. From Lemmas 3.5 and 3.4, for a sequence $r_{i} \rightarrow 0$, we may assume that $\hat{w}_{r_{i}}(\xi)$, converges to a bounded $n$-harmonic function $\hat{w}(\xi)$ in $A(0, \infty)=\mathbb{R}^{n} \backslash\{0\}$ (for some subsequence if necessary). When appying Lemma 3.5 and verifying $\mu_{i} \rightarrow 0$ in any compact subset of $\mathbb{R}^{n} \backslash\{0\}$, one needs to use (3.19) and Remark 3.1. Thanks to Liouville type theorem of Reshetnyak [55], 0 and $\infty$ are removable singularities of $\hat{w}(\xi)$ and $\hat{w}(\xi)=\hat{w}$ is a constant. Finally, one would like to use Lemma 3.6 in Sect. 3.3 to derive

$$
\hat{w}=\gamma^{-}=\liminf _{r \rightarrow 0} \frac{w(x)}{\log \frac{1}{|x|}}
$$

for any sequence $r_{i} \rightarrow 0$. The remaining issue is that all the sequential convergences are only the one weak in $W^{1, n}$ and strong in $W^{1, p}$ for any $1 \leq p<n$, which does not yet imply point-wise convergence as desired.

Now let us start with a proof of the uniqueness of $\hat{w}$ (i.e. (3.26)). Recall from (3.25) that

$$
\hat{w}_{r_{i}}\left(\xi_{r_{i}}\right) \rightarrow \gamma^{-}=\liminf _{r \rightarrow 0} \frac{w(x)}{\log \frac{1}{|x|}}
$$

for any sequence $r_{i} \rightarrow 0$. Since $\hat{w}_{r_{i}}(\xi)$ converges to $\hat{w}$ strongly in $W^{1, p}\left(A\left(r_{0}, \frac{1}{r_{0}}\right)\right), 1 \leq$ $p<n$ for any fixed small $r_{0}>0$, we know that

$$
\int_{B_{\frac{1}{2}}\left(\xi_{r_{i}}\right)}\left(\hat{w}_{r_{i}}(\xi)-\gamma^{-}\right)^{q} \rightarrow\left|B_{\frac{1}{2}}\left(\xi_{r_{i}}\right)\right|\left(\hat{w}-\gamma^{-}\right)^{q} \text { as } r_{i} \rightarrow 0
$$


for any $0<q<\infty$. By the way, $w_{r}(\xi) \geq \gamma^{-}$due to the definition of $\gamma^{-}$and Lemma 3.6. By invoking the weak Harnack inequality [39, Theorem 3.51]), we know

$$
\hat{w}_{r_{i}}\left(\xi_{r_{i}}\right)-\gamma^{-} \geq C\left(\int_{B_{\frac{1}{2}}\left(\xi_{r_{i}}\right)}\left(\hat{w}_{r_{i}}(\xi)-\gamma^{-}\right)^{q}\right)^{\frac{1}{q}}
$$

for any $\xi \in B_{\frac{1}{4}}\left(\xi_{r_{i}}\right)$ and some $0<q<\infty$. Clearly this would be a contradiction if $\hat{w} \neq \gamma^{-}$. So this finishes the proof of the uniqueness for sequential blow-down limits.

In the following, what we need to do is to refine the argument in the proof of Proposition 3.1 to show that, outside an $n$-thin set, the quotient $\frac{w(x)}{\log \frac{1}{|x|}}$ is not just bounded but actually convergent at the origin pointwisely. We will use the same notations and follow the same process. But we are in a better position than that we were in the proof of Proposition 3.1. First, we have the following improved (3.4) in Lemma 3.3

$$
\lim _{y \rightarrow 0} \inf _{x \in B\left(y, \frac{1}{\alpha}|y|\right)} \frac{w(x)}{\log \frac{1}{|x|}}=\gamma^{-} .
$$

This is because, from the uniqueness of all blow-down limits, we know

$$
\lim _{r \rightarrow 0} \hat{w}_{r}(\xi)=\gamma^{-}
$$

almost everywhere in $A_{r_{0}, \frac{1}{r_{0}}}$ and that $\hat{w}_{r}$ and $w_{r}$ only differ at the set $\tilde{E}$ that is $n$-thin at the origin. In fact we have the following, which is even more useful.

Lemma 3.7 Under the assumptions in Theorem 3.1.

$$
\lim _{y \rightarrow 0} \frac{\inf _{B(y, \alpha|y|)} w(x)}{\log \frac{1}{|y|}}=\gamma^{-}
$$

for any fixed $\alpha \in(0,1)$.

Proof First let

$$
\inf _{B(y, \alpha|y|)} \frac{w(x)}{\log \frac{1}{|x|}}=\frac{w\left(x_{0}\right)}{\log \frac{1}{\left|x_{0}\right|}}
$$

for some $x_{0} \in \bar{B}(y, \alpha|y|)$. Then $\frac{|y|}{\left|x_{0}\right|} \in\left[\frac{1}{1+\alpha}, \frac{1}{1-\alpha}\right]$ and

$$
\frac{\inf _{B(y, \alpha|y|)} w(x)}{\log \frac{1}{|y|}} \leq \frac{w\left(x_{0}\right)}{\log \frac{1}{|y|}}=\frac{w\left(x_{0}\right)}{\log \frac{1}{\left|x_{0}\right|}} \cdot \frac{\log \frac{1}{\left|x_{0}\right|}}{\log \frac{1}{|y|}} \leq \frac{w\left(x_{0}\right)}{\log \frac{1}{\left|x_{0}\right|}}\left(1+\frac{\log \frac{1}{1-\alpha}}{\log \frac{1}{|y|}}\right) .
$$

Next let

$$
\inf _{B(y, \alpha|y|)} w(x)=w\left(y_{0}\right)
$$

for some $y_{0} \in \bar{B}(y, \alpha|y|)$. Then $\frac{|y|}{\left|y_{0}\right|} \in\left[\frac{1}{1+\alpha}, \frac{1}{1-\alpha}\right]$ and

$$
\frac{\inf _{B(y, \alpha|y|)} w(x)}{\log \frac{1}{|y|}}=\frac{w\left(y_{0}\right)}{\log \frac{1}{|y|}}=\frac{w\left(y_{0}\right)}{\log \frac{1}{\left|y_{0}\right|}} \cdot \frac{\log \frac{1}{\left|y_{0}\right|}}{\log \frac{1}{|y|}} \geq \gamma^{-}\left(1+\frac{\log \frac{1}{1+\alpha}}{\log \frac{1}{|y|}}\right) .
$$

Therefore, squeezing from both sides, we derive (3.28). The proof is completed. 
Secondly, we apply [48, Theorem 1.6] to $w(y)-\inf _{B\left(y, \frac{3}{4}|y|\right)} w$ in $B\left(y, \frac{3}{4}|y|\right)$ and obtain

$$
w(y)-\inf _{B\left(y, \frac{3}{4}|y|\right)} w(x) \leq C_{2} \inf _{B\left(y, \frac{1}{4}|y|\right)}\left(w-\inf _{B\left(y, \frac{3}{4}|y|\right)} w\right)+C_{3} W_{1, n}^{\mu}\left(y, \frac{1}{2}|y|\right) .
$$

Hence,

$$
\frac{w(y)}{\log \frac{1}{|y|}} \leq \frac{\inf _{B\left(y, \frac{3}{4}|y|\right)^{w(x)}}}{\log \frac{1}{|y|}}+C_{2} \frac{\inf _{B\left(y, \frac{1}{4}|y|\right)^{w}}}{\log \frac{1}{|y|}}-C_{2} \frac{\inf _{B\left(y, \frac{3}{4}|y|\right)}{ }^{w}}{\log \frac{1}{|y|}}+C_{3} \frac{W_{1, n}^{\mu}\left(y, \frac{1}{2}|y|\right)}{\log \frac{1}{|y|}}
$$

which implies, by (3.28) in Lemma 3.7,

$$
\limsup _{y \rightarrow 0} \frac{w(y)}{\log \frac{1}{|y|}} \leq \gamma^{-}+C_{3} \limsup _{y \rightarrow 0} \frac{W_{1, n}^{\mu}\left(y, \frac{1}{2}|y|\right)}{\log \frac{1}{|y|}} .
$$

Thirdly, regarding the Wolff potential term in (3.30), we will also need an improved (3.9). For this purpose we first consider the convergent infinite series

$$
\sum_{i=1}^{\infty} \mu\left(\Omega_{i}\right) \leq 3 \mu(B(0,1))<\infty
$$

and use Paul du Bois-Reymond Theorem [16, (5) Page 40] (cf. [57]) to find a sequence $\zeta_{i} \rightarrow 0^{+}$as $i \rightarrow \infty$ such that

$$
\sum_{i=1}^{\infty} \frac{1}{\zeta_{i}} \mu\left(\Omega_{i}\right)<\infty
$$

for all $y \in A_{0,1}$. From the similar argument as in the proof of (3.9), we have,

$$
\operatorname{cap}_{n}\left(\left\{y \in B_{j}^{i}: W_{1, n}^{\mu}\left(y, \frac{1}{2}|y|\right)>\frac{\zeta_{i}^{\frac{1}{n-1}}}{C_{1}} \log \frac{1}{|y|}\right\}, 2 B_{j}^{i}\right) \leq \frac{C \frac{1}{\zeta_{i}} \mu\left(2 B_{j}^{i}\right)}{i^{n-1}} .
$$

Let

$E_{i j}=\left\{y \in B_{j}^{i}: W_{1, n}^{\mu}\left(y, \frac{1}{2}|y|\right)>\frac{\zeta_{i}^{\frac{1}{n-1}}}{C_{1}} \log \frac{1}{|y|}\right\} \bigcap \omega_{i}, E_{i}=\bigcup_{j} E_{i j}$, and $E=\bigcup_{i} E_{i}$.

Then (3.31) implies that

$$
\sum_{i=1}^{\infty} i^{n-1} \operatorname{cap}_{n}\left(E \bigcap \omega_{i}, \Omega_{i}\right) \leq \sum_{i} \frac{1}{\zeta_{i}} \mu\left(\Omega_{i}\right)<\infty,
$$

which says that $E$ is $n$-thin and

$$
\lim _{y \notin E \text { and } y \rightarrow 0} \frac{W_{1, n}^{\mu}\left(y, \frac{1}{2}|y|\right)}{\log \frac{1}{|y|}}=0 .
$$

Combining

$$
\frac{w(y)}{\log \frac{1}{|y|} \geq \gamma^{-}}
$$


with (3.30) and (3.32), we finally arrive at

$$
\lim _{y \notin E \text { and } y \rightarrow 0} \frac{w(y)}{\log \frac{1}{|y|}}=\gamma^{-}=\liminf _{y \rightarrow 0} \frac{w(y)}{\log \frac{1}{|y|}} .
$$

At last we will prove that, if $\left(B(0,2) \backslash\{0\}, e^{2 w}|d x|^{2}\right)$ is complete at the origin, then $m \geq 1$. Since

$$
\lim _{x \notin E, x \rightarrow 0} \frac{w(x)}{\log \frac{1}{|x|}}=m,
$$

if we can find a ray $P$ starting from 0 , such that $P \cap E \cap B\left(0, r_{0}\right)=\emptyset, 0<r_{0}<2$, then from the completeness, for any $\varepsilon>0$

$$
+\infty=\int_{P \cap B\left(0, r_{0}\right)} e^{w} d r \leq \int_{0}^{r_{0}} \frac{1}{r^{m+\varepsilon}} d r .
$$

So we know that $m+\varepsilon \geq 1$, which implies that $m \geq 1$. The question is reduced to finding such ray $P$ which has no intersection with the thin set $E$, at least in a small ball $B\left(0, r_{0}\right)$.

Define the projection map

$$
\begin{aligned}
\operatorname{Pr}: \omega(0,0) & \mapsto \partial B(0,1), \\
(r, \theta) & \rightarrow(1, \theta) .
\end{aligned}
$$

It is obvious a Lipschitz map, with Lipschitz constant 2. From the conformal invariance property of $n$-capacity, we know

$$
\operatorname{cap}_{n}(E \cap \omega(0, i), \Omega(0, i))=\operatorname{cap}_{n}\left(\left(2^{i} E\right) \cap \omega(0,0), \Omega(0,0)\right) .
$$

From the monotonicity property of capacity with respect to a Lipschitz map, $[1$, Theorem 5.2.1], we know

$$
\operatorname{cap}_{n}\left(\left(2^{i} E\right) \cap \omega(0,0), \Omega(0,0)\right) \geq \operatorname{cap}_{n}\left(\operatorname{Pr}\left(\left(2^{i} E\right) \cap \omega(0,0)\right), \Omega(0,0)\right) .
$$

From the thin property of $E$, we know

$$
\sum_{i} i^{n-1} \operatorname{cap}_{n}\left(\operatorname{Pr}\left(\left(2^{i} E\right) \cap \omega(0,0)\right), \Omega(0,0)\right)<+\infty .
$$

So

$$
\begin{aligned}
& \lim _{i_{0} \rightarrow+\infty} \operatorname{cap}_{n}\left(\cup_{i \geq i_{0}} \operatorname{Pr}\left(2^{i} E \cap \omega(0,0)\right), \Omega(0,0)\right) \\
& \quad \leq \lim _{i_{0} \rightarrow+\infty} \sum_{i \geq i_{0}} \operatorname{cap}_{n}\left(\operatorname{Pr}\left(2^{i} E \cap \omega(0,0)\right), \Omega(0,0)\right) \\
& \quad=0 .
\end{aligned}
$$

Since $\operatorname{cap}_{n}(\partial B(0,1), \Omega(0,0))$ is a positive number depending on $n$, so we can find a ray $P$ and $r_{0}>0$, such that $P \cap E \cap B\left(0, r_{0}\right)=\emptyset$. Thus the proof of Theorem 3.1 is completed. 


\section{Higher dimensional Taliaferro's estimates}

Let us start with Taliaferro's estimates in 2 dimensions.

Theorem 4.1 ([63, Theorem 2.1]) Suppose that $u$ is a $C^{2}$ positive solution to

$$
0 \leq-\Delta u \leq f(u)
$$

in a punctured neighborhood of the origin in $\mathbb{R}^{2}$, where $f:(0, \infty) \rightarrow(0, \infty)$ is a continuous function such that

$$
\log f(t)=O(t) \text { as } t \rightarrow \infty
$$

Then, either $u$ has a $C^{1}$ extension to the origin or

$$
\lim _{x \rightarrow 0} \frac{u(x)}{\log \frac{1}{|x|}}=m
$$

for some finite positive number $m$.

This can be viewed as the improvement of [6, Theorem 1.3], having no thin subset where the asymptotic behavior may differ from (4.1). Our next goal is to establish the higher dimensional analogue of [63, Theorem 2.1]. For the convenience of readers, we repeat our second main theorem in the introduction as follows:

Theorem 4.2 Let $w \in C^{2}(B(0,2) \backslash\{0\})$ be nonnegative and satisfy

$$
-\Delta_{n} w=f(x, w, \nabla w)
$$

in a punctured neighborhood of the origin in $\mathbb{R}^{n}$ and that

$$
\lim _{x \rightarrow 0} w(x)=+\infty,
$$

where $f$ is a nonnegative function satisfying

$$
0 \leq f(x, w, \nabla w) \leq C|\nabla w|^{n-2} e^{2 w}
$$

for some fixed constant $C$. Then

$$
\lim _{|x| \rightarrow 0} \frac{w(x)}{\log \frac{1}{|x|}}=m \geq 0
$$

and

$$
w(x) \geq m \log \frac{1}{|x|} \text { for } x \in B(0,1) \backslash\{0\} .
$$

Moreover, if $e^{2 w}|d x|^{2}$ is complete and non-compact at the origin, then $m \geq 1$.

Remark 4.1 The growth condition (4.3) can be replaced by

$$
0 \leq f(x, w, \nabla w) \leq C|\nabla w|^{p} e^{\alpha w}
$$

for any $p \in(0, n)$ and $\alpha>0$. This can be seen from (4.11) in the proof of Lemma 4.1 and (4.14) in the proof of Theorem 4.2. 


\subsection{The extension of Brezis-Merle inequality in higher dimensions}

From Theorem 2.4, we know, for some $\beta \geq 0$

$$
-\Delta_{n} w=\beta \delta_{0}+f(x, w, \nabla w)=\mu,
$$

where $f(x, w, \nabla w) \in L^{1}(B(0,1))$. The key analytic tool to remove the possibility of concentrating for solutions to $n$-Laplace equations like (2.18) and (4.2) with the critical growth condition (4.3) or more generally (4.5) is the higher dimensional analogue of the borderline Sobolev inequality established by Brezis and Merle in 2 dimensions in [15, Theorem 1], like Adams-Moser-Trudinger inequalities (please see $[32,44]$ and references therein). Our approach is different from [44] but the result is similar to that in [44]. To extend [15, Theorem 1] to general dimensions, we recall the Wolff potential

$$
W_{1, n}^{\mu}(x, r)=\int_{0}^{r} \mu(B(x, t))^{\frac{1}{n-1}} \frac{d t}{t}
$$

associated with a Radon measure $\mu$, and a Radon measure $\mu_{f}$ that is induced from a function $f \in L^{1}(\Omega)$

$$
\mu_{f}(U)=\int_{U \cap \Omega} f d x .
$$

Proposition 4.1 Let $\Omega \subset \mathbb{R}^{n}$ be a bounded domain with the diameter $D$. And let $f \in L^{1}(\Omega)$ be nonnegative. Then, for $\delta \in(0,1)$,

$$
\int_{\Omega} \exp \left(\frac{n(1-\delta) W_{1, n}^{\mu_{f}}(x, D)}{\|f\|_{L^{1}(\Omega)}^{\frac{1}{n-1}}}\right) d x \leq \frac{c(n) 2^{2 n+1}|B(0, D)|}{\delta^{n+1}}+2^{n}|\Omega| .
$$

Proof The proof is more or less standard in harmonic analysis. For the convenience of readers, we present a proof here. To start, we let $p>n-1$ and $\alpha^{p}=\mu_{f}(B(x, D))=\|f\|_{L^{1}(\Omega)} \leq 1$. Then

$$
\begin{aligned}
W_{1, n}^{\mu_{f}}(x, D) & \leq \int_{0}^{D} \mu_{f}(B(x, t))^{\frac{1}{p}} \frac{d t}{t} \\
& =\left.\mu(B(x, t))^{\frac{1}{p}} \log t\right|_{0} ^{D}+\int_{0}^{D} \log \frac{1}{t} d \mu(B(x, t))^{\frac{1}{p}} .
\end{aligned}
$$

Let

$$
M f(x)=\sup _{t>0} \frac{1}{|B(x, t)|} \int_{B(x, t) \cap \Omega} f(y) d y=\sup _{t>0} \frac{\mu(B(x, t))}{|B(x, t)|}
$$

be the Hardy-Littlewood maximal function of $f$. Hence

$$
\mu(B(x, t)) \leq M f(x)|B(0, t)|=n w_{n-1} t^{n} M f(x)
$$

almost everywhere, that is to say,

$$
\left.\mu(B(x, t))^{\frac{1}{p}} \log t\right|_{0} ^{D}=\alpha \log D
$$


almost everywhere. Therefore, by Jensen's inequality

$$
\begin{aligned}
\exp \left(W_{1, n}^{\mu}(x, D)\right) & \leq D^{\alpha} \int_{0}^{D} \frac{1}{t^{\alpha}} \frac{1}{\alpha} d \mu(B(x, t))^{\frac{1}{p}} \\
& \leq D^{\alpha}\left(\left.\frac{1}{\alpha} \frac{1}{t^{\alpha}} \mu(B(x, t))^{\frac{1}{p}}\right|_{0} ^{D}+\int_{0}^{D} \mu(B(x, t))^{\frac{1}{p}} \frac{1}{t^{\alpha+1}} d t\right) .
\end{aligned}
$$

If $\alpha<\frac{n}{p}$, then

$$
\begin{aligned}
\exp \left(W_{1, n}^{\mu}(x, D)\right) & \leq D^{\alpha}\left(D^{-\alpha}+\frac{1}{\frac{n}{p}-\alpha}\left(n w_{n-1}\right)^{\frac{1}{p}} M f(x)^{\frac{1}{p}} D^{\frac{n}{p}-\alpha}\right) \\
& =1+\frac{p}{n-\alpha p}|B(0, D)|^{\frac{1}{p}} M f(x)^{\frac{1}{p}}
\end{aligned}
$$

So we have, for $\lambda \geq 2$,

$$
\begin{aligned}
\left|\left\{x \in \Omega: \exp \left(W_{1, n}^{\mu}(x, D)\right) \geq \lambda\right\}\right| & \leq\left|\left\{x \in \Omega: M f(x) \geq \frac{(n-\alpha p)^{p} \lambda^{p}}{2^{p} p^{p}|B(0, D)|}\right\}\right| \\
& \leq \frac{c(n) 2^{p} p^{p}|B(0, D)|\|f\|_{L^{1}}}{(n-\alpha p)^{p} \lambda^{p}}
\end{aligned}
$$

thanks to the weak type Hardy-Littlewood maximal inequality. For $0<q<p$,

$$
\begin{aligned}
\int_{\Omega} \exp \left(q W_{1, n}^{\mu}(x, D)\right) d x & =\int_{0}^{+\infty}\left|\left\{x \in \Omega: \exp \left(W_{1, n}^{\mu}(x, D)\right) \geq t^{\frac{1}{q}}\right\}\right| d t \\
& \leq \int_{2^{q}}^{+\infty} \frac{c(n) 2^{p} p^{p}|B(0, D)|\|f\|_{L^{1}(\Omega)}}{(n-\alpha p)^{p} t^{\frac{p}{q}}} d t+\int_{0}^{2^{q}}|\Omega| d t \\
& \leq \frac{c(n) q 2^{q} p^{p}}{(p-q)(n-\alpha p)^{p}}|B(0, D)|\|f\|_{L^{1}(\Omega)}+2^{q}|\Omega| .
\end{aligned}
$$

Now consider $p=n\left(1-\frac{\delta}{2}\right), q=n(1-\delta), \delta \in(0,1)$, and $\alpha=\|f\|_{L^{1}(\Omega)}=1$ (otherwise one may consider $\bar{f}=\frac{f}{\|f\|_{L^{1}(\Omega)}}$ instead). Then, from (4.7), we have

$$
\int_{\Omega} \exp \left(n(1-\delta) \frac{W_{1, n}^{\mu}(x, D)}{\|f\|_{L^{1}(\Omega)}^{\frac{1}{n-1}}}\right) d x \leq \frac{c(n) 2^{2 n+1}|B(0, D)|}{\delta^{n+1}}+2^{n}|\Omega| .
$$

This finishes the proof.

\subsection{The uniform bound for the quotients}

In contrast to the proof of Theorem 3.1 in the previous subsection, we will be able to show, based on the growth condition (4.3) and Proposition 4.1, the quotient $\frac{w(x)}{\log \frac{1}{|x|}}$ is bounded: the analogue of [63, Theorem 2.3]. 
Lemma 4.1 Assume the same assumptions as in Theorem 4.2. Then the quotient

$$
\frac{w(x)}{\log \frac{1}{|x|}}
$$

is uniformly bounded in the punctured ball $B(0,1) \backslash\{0\}$.

Proof We prove Lemma 4.1 by contradiction. Assume otherwise, there is a sequence $\left\{x_{k}\right\}$ inside the punctured ball such that

$$
\frac{w\left(x_{k}\right)}{\log \frac{1}{\left|x_{k}\right|}} \rightarrow \infty \text { as }\left|x_{k}\right| \rightarrow 0
$$

One may consider the blow-up sequence

$$
v_{k}(\xi)=w\left(x_{k}+\frac{\left|x_{k}\right|}{4} \xi\right) \text { for } \xi \in B(0,2)
$$

and calculate

$$
\begin{aligned}
-\Delta_{n}^{\xi} v_{k} & =-\left(\frac{\left|x_{k}\right|}{4}\right)^{n} \Delta_{n}^{x} w\left(x_{k}+\frac{\left|x_{k}\right|}{4} \xi\right) \\
& =g_{k}(\xi) \leq C\left|x_{k}\right|^{2}\left|\nabla^{\xi} v_{k}\right|^{n-2} e^{2 v_{k}} \text { for } \xi \in B(0,2), \\
\int_{B(0,2)} g_{k}(\xi) d \xi & =\int_{B\left(x_{k}, \frac{\left|x_{k}\right|}{2}\right)} g(x) d x \rightarrow 0 \text { as } k \rightarrow \infty,
\end{aligned}
$$

where $-\Delta_{n} w=g+\beta \delta_{0}$ and $g \in L_{\text {loc }}^{1}(B(0,2))$ according to [9, Proposition 1.1]. We will argue in the similar way to that in [63]. We combine the non-linear potential theory [48, Theorem 1.6] with Lemma 3.3. For convenience, let us denote

$$
\lambda_{k}=\log \frac{1}{\left|x_{k}\right|} \rightarrow \infty \text { as } k \rightarrow \infty .
$$

Then it is implied from [48, Theorem 1.6] and Lemma 3.3 that

$$
\begin{aligned}
& \frac{1}{\lambda_{k}} W_{1, n}^{\mu g_{k}}(0,2) \rightarrow \infty \\
& g_{k}(\xi) \leq C\left|x_{k}\right|^{2}\left|\nabla^{\xi} v_{k}\right|^{n-2} e^{C_{1} \lambda_{k}+C_{2} W_{1, n}^{\mu g_{k}}(\xi, 2)} \text { for } \xi \in B(0,1) .
\end{aligned}
$$

Here $\mu_{g_{k}}$ is a measure such that $\mu_{g_{k}}(E)=\int_{E} g_{k} d \xi, E \subset B(0,2)$. A very important observation is that, when dealing with competing terms like $\lambda_{k}$ and $W_{1, n}^{\mu_{g_{k}}}(0,2)$, for

$$
\Omega_{k}=\left\{\xi \in B(0,1): W_{1, n}^{\mu_{g_{k}}}(\xi, 2) \geq \lambda_{k}\right\}
$$


we have

$$
\begin{aligned}
& \int_{\Omega_{k}}\left|g_{k}\right|^{\frac{n-1}{n-2}} d \xi \leq C\left|x_{k}\right|^{\frac{2(n-1)}{n-2}} \int_{\Omega_{k}}\left|\nabla^{\xi} v_{k}\right|^{n-1} e^{\frac{2(n-1)}{n-2} v_{k}} d \xi \\
& \leq C\left|x_{k}\right|^{\frac{2(n-1)}{n-2}} \int_{\Omega_{k}}\left|\nabla^{\xi} v_{k}\right|^{n-1} e^{\frac{2(n-1)}{n-2}\left(C_{1}(n) \inf _{B(0,1)} v_{k}+C_{2}(n) W_{1, n}^{\mu_{g_{k}}}(\xi, 2)\right)} d \xi \\
& \leq C\left|x_{k}\right|^{\frac{2(n-1)}{n-2}} \int_{B(0,1)}\left|\nabla^{\xi} v_{k}\right|^{n-1} e^{C_{3}(n) W_{1, n}^{\mu g_{k}}(\xi, 2)} d \xi \\
& \leq C\left|x_{k}\right|^{\frac{2(n-1)}{n-2}}\left(\int_{B(0,1)}\left|\nabla^{\xi} v_{k}\right|^{n-\frac{1}{2}} d \xi\right)^{\frac{2 n-2}{2 n-1}}\left(\int_{B_{2}(0)} e^{C_{4}(n) W_{1, n}^{\mu g_{k}}(\xi, 2)} d \xi\right)^{\frac{1}{2 n-1}} \\
& \leq C\left|x_{k}\right|^{\frac{2(n-1)}{n-2}-\frac{n-1}{2 n-1}} \\
& \left(\int_{B\left(x_{k}, \frac{\left|x_{k}\right|}{2}\right)}\left|\nabla^{x} w\right|^{n-\frac{1}{2}} d x\right)^{\frac{2 n-2}{2 n-1}}\left(\int_{B_{2}(0)} e^{C_{4}(n) W_{1, n}^{\mu_{g_{k}}(\xi, 2)}} d \xi\right)^{\frac{1}{2 n-1}} \leq C .
\end{aligned}
$$

Make a note that $\frac{2(n-1)}{n-2}-\frac{n-1}{2 n-1}>1$. The last step in the above inequalities relies on Proposition 4.1 and the $L^{p}$-gradient estimates for $n$-superharmonic functions for any $p<n$ (for example by Theorem 2.4). This implies that

$$
\mu_{g_{k}}\left(B(0, t) \cap \Omega_{k}\right) \leq C t^{\frac{n}{n-1}}
$$

for some positive constant $C>0$. Observe that

$$
\mu_{g_{k}}(B(0, t))^{\frac{1}{n-1}} \leq \mu_{g_{k}}\left(B(0, t) \cap \Omega_{k}\right)^{\frac{1}{n-1}}+\mu_{g_{k}}\left(B(0, t) \backslash \Omega_{k}\right)^{\frac{1}{n-1}}
$$

which implies

$$
W_{1, n}^{\mu_{g_{k}}}(0,2) \leq C+C \int_{0}^{2} \mu_{g_{k}}\left(B(0, t) \backslash \Omega_{k}\right)^{\frac{1}{n-1}} \frac{d t}{t} .
$$

To estimate the second term on the right side of the above equation, one notices that, for $\xi \in B(0,1) \backslash \Omega_{k}$,

$$
g_{k}(\xi) \leq C\left|x_{k}\right|^{2}\left|\nabla^{\xi} v_{k}\right|^{n-2} e^{C_{2}(n) \lambda_{k}+C_{3}(n) W_{1, n}^{\mu_{g_{k}}(\xi, 2)}} \leq C\left|x_{k}\right|^{2}\left|\nabla^{\xi} v_{k}\right|^{n-2} e^{C_{5}(n) \lambda_{k}}
$$


from (4.10). Therefore

$$
\begin{aligned}
\int_{B(0, t) \backslash \Omega_{k}} g_{k}(\xi) d \xi & \leq C \int_{B(0, t) \backslash \Omega_{k}}\left|x_{k}\right|^{2}\left|\nabla^{\xi} v_{k}\right|^{n-2} e^{C_{5}(n) \lambda_{k}} d \xi \\
& \leq C\left|x_{k}\right|^{2-\frac{n-2}{n-1}} e^{C_{5}(n) \lambda_{k}} \int_{B(0, t) \backslash \Omega_{k}}\left|x_{k}\right|^{\frac{n-2}{n-1}}\left|\nabla^{\xi} v_{k}\right|^{n-2} d \xi \\
& \leq C\left|x_{k}\right|^{2-\frac{n-2}{n-1}} e^{C_{5}(n) \lambda_{k}} t^{\frac{n}{n-1}}\left(\int_{B(0, t) \backslash \Omega_{k}}\left(\left|x_{k}\right|^{\frac{n-2}{n-1}}\left|\nabla^{\xi} v_{k}\right|^{n-2}\right)^{\frac{n-1}{n-2}} d \xi\right)^{\frac{n-2}{n-1}} \\
& \leq C\left|x_{k}\right|^{2-\frac{n-2}{n-1}} e^{C_{5}(n) \lambda_{k}} t^{\frac{n}{n-1}}\left(\int_{B^{x}(0,1)}\left|\nabla^{x} w\right|^{n-1} d x\right)^{\frac{n-2}{n-1}}
\end{aligned}
$$

We now calculate separately, for $\rho_{k}$ to be fixed next,

$$
\begin{aligned}
\int_{0}^{2} \mu_{g_{k}}\left(B(0, t) \backslash \Omega_{k}\right)^{\frac{1}{n-1}} \frac{d t}{t} & =\int_{0}^{\rho_{k}} \mu_{g_{k}}\left(B(0, t) \backslash \Omega_{k}\right)^{\frac{1}{n-1}} \frac{d t}{t}+\int_{\rho_{k}}^{2} \mu_{g_{k}}\left(B(0, t) \backslash \Omega_{k}\right)^{\frac{1}{n-1}} \frac{d t}{t} \\
& \leq(n-1)^{2} C\left|x_{k}\right|^{2} e^{C_{5}(n) \lambda_{k}} \rho_{k}^{\frac{1}{(n-1)^{2}}}+C \log \frac{1}{\rho_{k}}+C .
\end{aligned}
$$

Let us fix

$$
\rho_{k}=e^{-(n-1)^{2} C_{5}(n) \lambda_{k}} \in(0,2) .
$$

We thus get

$$
\int_{0}^{2} \mu_{g_{k}}\left(B(0, t) \backslash \Omega_{k}\right)^{\frac{1}{n-1}} \frac{d t}{t} \leq C+C \lambda_{k}
$$

which contradicts with (4.9) in the light of (4.12). So Lemma 4.1 is proved.

\subsection{The proof of Theorem $\mathbf{4 . 2}$}

Lemma 4.1 enables us to proceed with blow-down argument without going through Sects. 3.1 and 3.2. We are now ready to prove Theorem 4.2.

The proof of Theorem 4.2 We again consider the blow-down

$$
w_{r}(\xi)=\frac{w(r \xi)}{\log \frac{1}{r}}
$$

and calculate that, from Lemma 4.1,

$$
\left|w_{r}(\xi)\right| \leq C \frac{\log \frac{1}{r}+\left|\log \frac{1}{|\xi|}\right|}{\log \frac{1}{r}} \leq 2 C \text { for all } \xi \in A_{r, \frac{1}{r}}=\left\{\xi \in \mathbb{R}^{n}:|\xi| \in\left(r, \frac{1}{r}\right)\right\}
$$


From here, similar to the approach of the proof of Theorem 3.1 in the previous section, one may complete the proof of Theorem 4.2. To do so, we continue to use the notation

$$
\gamma^{-}=\liminf _{x \rightarrow 0} \frac{w(x)}{\log \frac{1}{|x|}}
$$

as in (3.26) in Sect. 3.4.

First, as in the previous section, one may prove that on $\mathbb{R}^{n} \backslash\{0\}, w_{r}(\xi)$ converges to $\gamma^{-}$in $W_{l o c}^{1, n}$ weakly and $W_{l o c}^{1, p}$ strongly for any $p<n$, which implies that $w_{r}(\xi)$ converges to $\gamma^{-}$ pointwisely almost everywhere. This heavily relies on the uniqueness of sequential blowdown limits established in the proof Theorem 3.1 in Sect. 3.4. Hence we want to improve from here that $\frac{w(x)}{\log \frac{1}{|x|}}$ converges to $\gamma^{-}$pointwisely as we did in the proof Theorem 3.1 in Sect. 3.4. In the light of (3.33) and (3.30), we need to show (3.32) with no thin set $E$ excluded, i.e.

$$
\lim _{x \rightarrow 0} \frac{W_{1, n}^{\mu}\left(x, \frac{1}{2}|x|\right)}{\log \frac{1}{|x|}}=0 .
$$

To prove (4.13), we recall that

$$
W_{1, n}^{\mu}\left(x, \frac{1}{2}|x|\right)=\int_{0}^{\frac{1}{2}|x|} \mu(B(x, s))^{\frac{1}{n-1}} \frac{d s}{s}
$$

where

$$
\mu(B(x, s))=\int_{B(x, s)} f(x, w, \nabla w) d x \leq C \int_{B(x, s)}|\nabla w|^{n-2} e^{2 w} d x .
$$

From (4.8) and [9, Proposition 1.1], we know that $\mu(B(0,1))<\infty$ and that $\mu(B(x, s)) \rightarrow 0$ as $s \rightarrow 0$ for $s \leq \frac{1}{2}|x|$. But one needs a sharper asymptotics on the rate at which $\mu(B(x, s)) \rightarrow$ 0 as $s \rightarrow 0$ when calculating the Wolff potential. Therefore we recall [48, Theorem 1.6]

$$
w(x) \leq C_{2} \inf _{B\left(x, \frac{1}{4}|x|\right)} w+C_{3} W_{1, n}^{\mu}\left(x, \frac{1}{2}|x|\right)
$$

and estimate,

$$
\mu(B(x, s)) \leq C|x|^{-2 C_{2}\left(\gamma^{-}+1\right)} \int_{B(x, s)}|\nabla w|^{n-2} e^{2 C_{3} W_{1, n}^{\mu}\left(x, \frac{1}{2}|x|\right)} d x .
$$

Applying Hölder's inequality, we have

$$
\begin{aligned}
& \mu(B(x, s)) \leq C|x|^{-2 C_{2}\left(\gamma^{-}+1\right)} \\
& \left(\int_{B(x, s)}|\nabla w|^{n-1} d x\right)^{\frac{n-2}{n-1}}\left(\int_{B(x, s)} e^{2(n-1) C_{3} W_{1, n}^{\mu}\left(x, \frac{1}{2}|x|\right)} d x\right)^{\frac{1}{n-1}}
\end{aligned}
$$


Then, we use Proposition 4.1 and derive

$$
\mu(B(x, s)) \leq C|x|^{-2 C_{2}\left(\gamma^{-}+1\right)}\left(\int_{B(x, s)}|\nabla w|^{n-1} d x\right)^{\frac{n-2}{n-1}}
$$

Finally, we use $L^{p}$ bound for the gradient of the $n$-superharmonic function $w$ for $p=n-\frac{1}{2}<$ $n$ and get

$$
\begin{aligned}
\mu(B(x, s)) & \leq C|x|^{-2 C_{2}\left(\gamma^{-}+1\right)}\left(\int_{B(x, s)}|\nabla w|^{n-\frac{1}{2}} d x\right)^{\frac{n-2}{n-\frac{1}{2}}} s^{\frac{n(n-2)}{2(n-1)\left(n-\frac{1}{2}\right)}} \\
& \leq C|x|^{-2 C_{2}\left(\gamma^{-}+1\right)} s^{\frac{n(n-2)}{2(n-1)\left(n-\frac{1}{2}\right)}}
\end{aligned}
$$

Here we are indifferent to constants except maybe those from [48, Theorem 1.6]. Therefore, going back to estimate the Wolff potential, we have

$$
\begin{aligned}
W_{1, n}^{\mu}\left(x, \frac{1}{2}|x|\right) & =\int_{0}^{\rho} \mu(B(x, s))^{\frac{1}{n-1}} \frac{d s}{s}+\int_{\rho}^{\frac{1}{2}|x|} \mu(B(x, s))^{\frac{1}{n-1}} \frac{d s}{s} \\
& \leq C|x|^{-\frac{2 C_{2}\left(\gamma^{-}+1\right)}{n-1}} \rho^{\frac{n(n-2)}{2(n-1)^{2}\left(n-\frac{1}{2}\right)}}+o(1) \log \frac{1}{\rho} .
\end{aligned}
$$

Taking

$$
\rho=|x| \frac{4 C_{2}\left(\gamma^{-}+1\right)(n-1)\left(n-\frac{1}{2}\right)}{n(n-2)}
$$

and noting that $o(1)$ is with respect to $x \rightarrow 0$ implies (4.13). So (4.4) is established. It is then easily seen that

$$
\int_{0}^{1} e^{w} d r=\infty
$$

implies $m \geq 1$ from (4.4). Thus the proof is completed.

\section{Locally conformally flat manifolds}

In this section we are going to use the property of $n$-superharmonic functions to study the asymptotic behavior at the end of a complete locally conformally flat manifold $\left(M^{n}, g\right)$. Based on the injectivity of the development maps of [58, Theorem 4.5], in [67, Theorem 1] and later in [18], the following classification result was shown.

Theorem 5.1 ([18,67]) Let $\left(M^{n}, g\right)$ be a complete conformally flat manifold of dimension $n \geq 3$ with nonnegative Ricci curvature. Then

- $M$ is globally conformally equivalent to $\mathbb{R}^{n}$;

- $M$ is globally conformally equivalent to a spaceform of positive curvature; 
- $M$ is locally isometric to the cylinder $\mathbb{R} \times \mathbb{S}^{n-1}$;

- $M$ is isometric to a complete flat manifold.

We confine ourselves to the first case in the above classification theorem. Recall that, on $\left(\mathbb{R}^{n}, e^{2 \phi}|d x|^{2}\right)$, in the light of (2.18),

$$
-\Delta_{n} \phi=\operatorname{Ric}_{g}\left(\nabla^{g} \phi\right)|\nabla \phi|^{n-2} e^{2 \phi},
$$

where $\operatorname{Ric}_{g}\left(\nabla^{g} \phi\right)$ is the Ricci curvature of the conformal metric $g=e^{2 \phi}|d x|^{2}$ in the $\nabla^{g} \phi$ direction. As a consequence of Theorems 3.1 and 4.2, for a globally conformally flat manifold $\left(\mathbb{R}^{n}, e^{2 \phi}|d x|^{2}\right.$ ), we therefore are able to deduce our third main theorem stated in the introduction. For the convenience of readers, we repeat it as follows:

Theorem 5.2 Suppose that $\left(\mathbb{R}^{n}, e^{2 \phi}|d x|^{2}\right)$ is complete with nonnegative Ricci $(n \geq 3)$, where $\phi$ is a smooth function. Then there is a subset $E \subset \mathbb{R}^{n}$, which is $n$-thin at infinity, such that

$$
\lim _{x \notin E \rightarrow \infty} \frac{\phi(x)}{\log \frac{1}{|x|}}=\liminf _{x \rightarrow \infty} \frac{\phi(x)}{\log \frac{1}{|x|}}=m
$$

and

$$
\phi(x) \geq m \log \frac{1}{|x|}-C
$$

for some constant $C$, where

$$
m|m|^{n-2}=\frac{1}{w_{n-1}} \int_{\mathbb{R}^{n}} \operatorname{Ric}_{g}\left(\nabla^{g} \phi\right)|\nabla \phi|^{n-2} e^{2 \phi} d x .
$$

Moreover,

- $m \in[0,1]$ and $m=0$ if and only if $g$ is flat, i.e. $\phi(x)$ is a constant function;

- if Ric is $_{\text {bounded, then }}$

$$
\lim _{x \rightarrow \infty} \frac{\phi(x)}{\log \frac{1}{|x|}}=\liminf _{x \rightarrow \infty} \frac{\phi(x)}{\log \frac{1}{|x|}}=m .
$$

We remark that Theorem 5.2 should be compared with [7,23,25]. In [7] it was proved that, a complete noncompact manifold $\left(M^{n}, g\right)$ satisfying

$$
\begin{aligned}
\text { Ric } & \geq 0 \\
\operatorname{vol}(B(0, r)) & \geq \gamma r^{n} \text { for some } \gamma>\frac{1}{2} w_{n-1} \\
|\mathrm{Rm}| & \leq C r^{-2}
\end{aligned}
$$

and in addition,

$$
\text { either }|\mathrm{Rm}|=o\left(r^{-2}\right) \text { or } \int_{M}|\mathrm{Rm}|^{\frac{n}{2}} d v o l<\infty,
$$

is actually isometric to the Euclidean space. The assumption of $\gamma>\frac{1}{2} w_{n-1}$ is essential, in light of Eguchi-Hanson metrics. In [25], Colding proved remarkably that a complete manifold with nonnegative Ricci curvature is isometric to the Euclidean space, if one tangent 
cone at infinity is the Euclidean space. In [23], on the other hand, it was proved, a complete noncompact conformally flat manifold with nonnegative Ricci and satisfying

$$
\frac{1}{\operatorname{vol}\left(B\left(x_{0}, r\right)\right)} \int_{B\left(x_{0}, r\right)} \text { Rdvol }=o\left(r^{-2}\right)
$$

where the scalar curvature $R$ is bounded, is actually isometric to the Euclidean space. The comparison of Theorem 5.2 to the rigidity results in $[7,23,25]$ would be more direct if the intrinsic distance function $r$ on the manifold and $|x|^{1-m}$ in Euclidean space as the background metric are equivalent, which seems to require something stronger than (5.4).

The proof of Theorem 5.2 First we use the inversion to turn the asymptotic problem to be the one at around the origin as those studied in Theorems 3.1 and 4.2. Let

$$
w(y)=\phi\left(\frac{y}{|y|^{2}}\right)-2 \log |y|
$$

for $y \in \mathbb{R}^{n} \backslash\{0\}$. Then $g=e^{2 \phi(x)}|d x|^{2}=e^{2 w(y)}|d y|^{2}$, where $x=\frac{y}{|y|^{2}}$. Then from (2.18) we know

$$
-\Delta_{n}^{y} w(y)=\operatorname{Ric}_{g}\left(\nabla^{g} w\right)|\nabla w|^{n-2} e^{2 w} .
$$

Because $g=e^{2 w}|d y|^{2}$ is complete at the origin and its scalar curvature $R \geq 0$, from [20, Proposition 8.1], we know that

$$
\lim _{y \rightarrow 0} w(y)=+\infty .
$$

Hence, from Theorems 2.4 and 3.1, we know there are a number $m_{1} \geq 1$ and a set $E_{1}$, which is $n$-thin at 0 such that,

$$
\lim _{y \notin E_{1}, y \rightarrow 0} \frac{w(y)}{\log \frac{1}{|y|}}=m_{1}
$$

and $w(y) \geq m_{1} \log \frac{1}{|y|}-C$. Now, translating these back to $\phi(x)$ through the inversion, we have

$$
\begin{gathered}
\phi(x) \geq-m \log |x|-C \text { for any }|x| \text { large } \\
\phi(x) \leq-m \log |x|+o(\log |x|) \text { for any }|x| \text { large and outside of a set } E,
\end{gathered}
$$

where $m=2-m_{1} \leq 1$ and $E=\left\{x ; \frac{x}{|x|^{2}} \in E_{1}\right\}$. Moreover, from Definition 3.1, we know $E$ is $n$-thin at infinity. So (5.1) is proved.

If, in addition, Ricci curvature is bounded, then

$$
\operatorname{Ric}_{g}\left(\nabla^{g} w\right)|\nabla w|^{n-2} e^{2 w} \leq C\left|\nabla^{y} w\right|^{n-2} e^{2 w}
$$

and (5.4) follows from Theorem 4.2. Assume (5.4) holds. Then it is obvious that $m \in[0,1]$. If (5.3) holds, then it is obvious that $m \geq 0$, and if equality holds, then $\operatorname{Ric}_{g}\left(\nabla^{g} w\right)|\nabla w|^{n-2} e^{2 w}$ must be identically 0 , which implies that $\phi(x)$ is an $n$-harmonic function on $\mathbb{R}^{n}$, which is lower bounded by a constant from (5.2). So $\phi$ has to be a constant in this case due to [39, Theorem 6.2 and Corollary 6.11].

To finish the proof of Theorem 5.2, it suffices to prove (5.3). To do so, we are going to integrate

$$
\int_{\Omega} \operatorname{Ric}_{g}\left(\nabla^{g} \phi\right)|\nabla \phi|^{n-2} e^{2 \phi} d x=\int_{\Omega}\left(-\Delta_{n} \phi\right) d x=-\int_{\partial \Omega}|\nabla \phi|^{n-2} \frac{\partial \phi}{\partial \mathbf{n}} d S_{x} .
$$


To avoid relying on sharp gradient estimates for $\phi$ on the boundary of any exhausting family of domains $\Omega$ in $\mathbb{R}^{n}$, we will work with chosen exhausting families of domains. Our construction of the exhausting families of domains is very natural and desirable. Let us define, for $m \in \mathbb{R}$ and a positive small number $\varepsilon$ and a positive large number $t$,

$$
\begin{aligned}
& G_{\varepsilon, t}^{+}(x)=-(m+\varepsilon) \max \{\log |x|, 0\}+t, \\
& G_{\varepsilon, t}^{-}(x)=-(m-\varepsilon) \max \{\log |x|, 0\}-t .
\end{aligned}
$$

And let

$\Omega_{\varepsilon, t}^{+}=$the connected component of $\left\{x: G_{\varepsilon, t}^{+}(x)>\phi(x)\right\}$ that includes the origin,

$\Omega_{\varepsilon, t}^{-}=$the connected component of $\left\{x: G_{\varepsilon, t}^{-}(x)<\phi(x)\right\}$ that includes the origin.

Claim For a fixed $\varepsilon>0$, there is a sequence of positive number $t_{k} \rightarrow \infty$ such that the collection $\left\{\Omega_{\varepsilon, t_{k}}^{+}\right\}$is an exhausting family of smooth and bounded domains for $\mathbb{R}^{n}$. Similarly, for a fixed $\varepsilon>0$, there also exists a sequence of positive numbers $s_{k} \rightarrow \infty$ such that the collection $\left\{\Omega_{\varepsilon, s_{k}}^{-}\right\}$is an exhausting family of smooth and bounded domains for $\mathbb{R}^{n}$.

Proof of Claim Let us first consider $\Omega_{\varepsilon, t}^{+}$. Smoothness is not a problem, one can always perturb and get the smooth ones. From the definition, it is easily seen that, for any fixed $R$,

$$
B(0, R) \subset \Omega_{\varepsilon, t}^{+}
$$

whenever $t$ is sufficiently large. Hence $\Omega_{\varepsilon, t}^{+}$can exhaust the entire space. Meanwhile, for each fixed $\varepsilon$ and $t, \Omega_{\varepsilon, t}^{+}$is bounded in the light of (5.5).

Let us turn to $\Omega_{\varepsilon, t}^{-}$. The only issue different is the boundedness for $\Omega_{\varepsilon, t}^{-}$when $\varepsilon$ and $t$ are arbitrarily fixed. It is easily seen that each $\Omega_{\varepsilon, t}^{-} \backslash E$ is bounded, because of (5.6). Then $\Omega_{\varepsilon, t}^{-}$ is the connected component that includes the origin and $n$-thin at infinity. Let $\gamma$ be a ray in Euclidean space starting from the origin. If $\Omega_{\varepsilon, t}^{-}$is not bounded, then again from $[1$, Theorem 5.2.1], we know for $i$ arbitrarily large,

$$
\operatorname{cap}\left(\Omega_{\varepsilon, t}^{-} \cap \omega(i, \infty), \Omega(i, \infty)\right) \geq C \operatorname{cap}(\gamma \cap \omega(i, \infty), \Omega(i, \infty)) \geq C(n)>0,
$$

since the map from $\Omega_{\varepsilon, t}^{-}$to $\gamma$ which preserves the radius is a Lipschitz map. Now we get a contradiction with the fact $E$ is $n$-thin at infinity. So the proof of this claim is finished.

Remark 5.1 In the above proof, [1, Theorem 5.2.1] can be replaced by the following lemma.

Lemma 5.1 ([56, Lemma 1.4 page 212] and [33, Theorem 4]) Let $K=(A, B)$ be a condenser in Euclidean $n$-space, where both $A$ and $B$ are connected. Assume that

(1) A is outside the unit ball, unbounded and includes a point on the unit sphere;

(2) B includes the origin and has a point with length $L$. Then

$$
\operatorname{cap}_{n}(B, A) \geq \frac{c_{n}}{\left(\log \left(1+\frac{1}{L}\right)\right)^{n-1}} .
$$

Note that in our case $A=\Omega(i, \infty)^{c}$ or $\Omega(i, 0)^{c}$ is unbounded. We will use this estimate in the next section.

Now we return to the proof of Theorem 5.2. On $\partial \Omega_{\varepsilon, t}^{+}$, we want

$$
|\nabla \phi|^{n-2} \frac{\partial \phi}{\partial \mathbf{n}} \geq\left|\nabla G_{\varepsilon, t}^{+}\right|^{n-2} \frac{\partial G_{\varepsilon, t}^{+}}{\partial \mathbf{n}} .
$$


This is because, in the normal direction at each point $x \in \partial \Omega_{\varepsilon, t}^{+}$,

$$
\frac{\partial \phi}{\partial \mathbf{n}}(x) \geq \frac{\partial G_{\varepsilon, t}^{+}}{\partial \mathbf{n}}(x)
$$

due to the definition of $\Omega_{\varepsilon, t}$. While, obviously, in the direction $\tau$ tangent to the boundary at each $x \in \partial \Omega_{\varepsilon, t}^{+}$,

$$
\frac{\partial \phi}{\partial \emptyset}(x)=\frac{\partial G_{\varepsilon, t}^{+}}{\partial \emptyset}(x) .
$$

Therefore

- if $\frac{\partial G_{\varepsilon, t}^{+}}{\partial \mathbf{n}}(x) \geq 0$, then we have $|\nabla \phi(x)| \geq\left|\nabla G_{\varepsilon, t}^{+}(x)\right|$ and (5.9) holds;

- if $\frac{\partial G_{\varepsilon, t}^{+}}{\partial \mathbf{n}}(x)<0$ and $\frac{\partial \phi}{\partial \mathbf{n}}(x) \geq 0,(5.9)$ trivially holds;

- if $\frac{\partial G_{\varepsilon, t}^{+}}{\partial \mathbf{n}}(x) \leq \frac{\partial \phi}{\partial \mathbf{n}}(x)<0$, then $|\nabla \phi(x)| \leq\left|\nabla G_{\varepsilon, t}^{+}(x)\right|$ and still (5.9) holds.

So (5.9) always holds as desired. Therefore, continuing from (5.7),

$$
\begin{aligned}
\int_{\Omega_{\varepsilon, t}^{+}} \operatorname{Ric}_{g}\left(\nabla^{g} \phi\right)|\nabla \phi|^{n-2} e^{2 \phi} d x & =-\int_{\partial \Omega_{\varepsilon, t}^{+}}|\nabla \phi|^{n-2} \frac{\partial \phi}{\partial \mathbf{n}} d S \\
& \leq-\int_{\partial \Omega_{\varepsilon, t}^{+}}\left|\nabla G_{\varepsilon, t}^{+}\right|^{n-2} \frac{\partial G_{\varepsilon, t}^{+}}{\partial \mathbf{n}} d S \\
& =(m+\varepsilon)|m+\varepsilon|^{n-2} w_{n-1} .
\end{aligned}
$$

Here in the last step, we use the fact that

$$
-\int_{\partial \Omega_{\varepsilon, t}^{+}}\left|\nabla \tilde{G}_{\varepsilon, t}^{+}\right|^{n-2} \frac{\partial \tilde{G}_{\varepsilon, t}^{+}}{\partial \mathbf{n}} d S=\int_{\Omega_{\varepsilon, t}^{+}}\left(-\Delta \tilde{G}_{\varepsilon, t}^{+}\right) d x=(m+\varepsilon)|m+\varepsilon|^{n-2} w_{n-1},
$$

for $t$ very large, where

$$
\tilde{G}_{\varepsilon, t}^{+}=-(m+\varepsilon) \log |x|+t
$$

which agrees with $G_{\varepsilon, t}^{+}$outside the unit ball.

Similarly, using $G_{\varepsilon, t}^{-}$and $\Omega_{\varepsilon, t}^{-}$, we have

$$
\int_{\Omega_{\varepsilon, t}^{-}} \operatorname{Ric}_{g}\left(\nabla^{g} \phi\right)|\nabla \phi|^{n-2} e^{2 \phi} d x \geq(m-\varepsilon)|m-\varepsilon|^{n-2} w_{n-1} .
$$

Thus, by the exhaustion property of the chosen families of domains, (5.3) follows. The proof of Theorem 5.2 is completed.

\section{Hypersurfaces in hyperbolic space}

In this section we want to use Theorems 3.1 and 4.2 to study the asymptotic end structure of embedded hypersurfaces in hyperbolic space with nonnegative Ricci. Our work here is inspired by and improves the results in $[3,4]$. In the light of [12, Main Theorem], in this paper, 
we focus on the study of end structure at infinity for these hypersurfaces in hyperbolic space with nonnegative Ricci and one single end. We refer readers to Sect. 2.5.2 for a very brief introduction of complete and globally strictly convex hypersurfaces in hyperbolic space (cf. $[3,4,11,12])$. For convenience of readers, we first recall Busemann coordinates in hyperbolic space. We start with half space model for hyperbolic space

$$
R_{+}^{n+1}=\left\{\left(x_{1}, x_{2}, \ldots, x_{n}, x_{n+1}\right):\left(x_{1}, x_{2}, \ldots, x_{n}\right) \in \mathbb{R}^{n} \text { and } x_{n+1}>0\right\}
$$

with the hyperbolic metric

$$
g_{\mathbb{H}}=\frac{|d x|^{2}+\left|d x_{n+1}\right|^{2}}{x_{n+1}^{2}} .
$$

We use the notation that $\partial_{\infty} \mathbb{H}^{n+1}=\mathbb{R}^{n} \bigcup\left\{p_{\infty}\right\}$ in this half space model. A vertical graph in hyperbolic space is the hypersurface given by

$$
\phi(x)=(x, f(x)): \Omega \rightarrow \mathbb{R}_{+}^{n+1}, x=\left(x_{1}, \cdots, x_{n}\right)
$$

for a function

$$
x_{n+1}=f(x): \Omega \subset \mathbb{R}^{n} \rightarrow \mathbb{R}_{+}=\{s \in \mathbb{R}: s>0\} .
$$

The Busemann coordinates is $(x, \rho) \in \mathbb{R}^{n} \times \mathbb{R}$ such that

$$
\rho=\log x_{n+1} .
$$

In this coordinates

$$
g_{\mathbb{H}}=e^{-2 \rho}|d x|^{2}+d \rho^{2} .
$$

Therefore the height function for a vertical graph in Busemann coordinates is

$$
\rho(x)=\log f(x): \Omega \rightarrow \mathbb{R} .
$$

It is worth to mention that, in such coordinates, an equidistant hypersurface with one end at $p_{\infty}$ is represented by

$$
\rho=\log \left|x-x_{0}\right|+C
$$

for the other end at some point $x_{0} \in \mathbb{R}^{n} \subset \partial_{\infty} \mathbb{H}^{n+1}$ and a constant $C$.

For a vertical graph $\rho=\rho(x)$ in Busemann coordinates, one considers the inner rotation hypersurface as in $[3,4]$. More precisely, let

$$
\hat{\rho}(r)=\sup _{|x|=r} \rho(x) .
$$

Then the vertical graph $\rho=\hat{\rho}(|x|)$ is the inner rotation hypersurface to the graph $\rho=\rho(x)$. Similar to what was observed in [3,4], using inner rotation hypersurfaces, we first observe:

Lemma 6.1 Suppose that a hypersurface $\Sigma \subset \mathbb{H}^{n+1}$ is a vertical graph of $\rho=\rho(x)$ over $\Omega \subset \mathbb{R}^{n}$ in Busemann coordinates. And suppose that $\Sigma$ has nonnegative Ricci curvature and is complete with one end at $p_{\infty}$. Then $\Omega=\mathbb{R}^{n}$ and there is an equidistant hypersurface as the graph of $\rho=\log |x|+C$ such that

$$
\rho(x) \leq \hat{\rho}(|x|) \leq \log |x|+C
$$

for all $|x|$ sufficiently large. In other words, at least at the end, the hypersurface $\Sigma$ is under an equidistant hypersurface. 
Proof First of all, we know the hypersurface is globally and strictly convex. Let $\hat{\Sigma}$ be the inner rotation hypersurface as the graph of $\rho=\hat{\rho}(r)$ defined in (6.1), which is above the given hypersurface $\Sigma$ as the graph of $\rho=\rho(x)$. It is easy to see that $\partial_{\infty} \hat{\Sigma}=\partial_{\infty} \Sigma=p_{\infty}$.

Applying the maximum principle to $\rho$, we can prove that $\hat{\rho}$ is non-decreasing. From the fact that $A \log r+B$ is $n$-harmonic and the maximum principle, following the argument of [37, Page 66], we can show that $\hat{\rho}$ is convex in $\log r$. Hence $\hat{\rho}$ is continuous and differentiable except at countably many points. Moreover, at a singular point $a, \hat{\rho}_{-}^{\prime}(a)<\hat{\rho}_{+}^{\prime}(a)$. When $\hat{\rho}$ is differentiable for $r \in(a, b)$, the corresponding portion of $\hat{\Sigma}$ has nonnegative Ricci curvature. Because, for any fixed $r \in(a, b), \hat{\Sigma}$ is supported by $\Sigma$ at least at some point $x$ with $|x|=r$. By the comparison of principal curvatures, one may easily derive that the Ricci curvature of $\hat{\Sigma}$ is nonnegative from that the Ricci curvature of $\Sigma$ is nonnegative. Therefore $\hat{\Sigma}$ has Ricci curvature nonnegative everywhere on the regular part of $\hat{\Sigma}$.

Now let us assume without loss of any generality that $0 \in \Omega$. Let $R$ be the radius of the maximal ball $B(0, R) \subset \Omega$. For any fixed $r_{0}<R$, we take $C$ sufficiently large such that

$$
\hat{\rho}\left(r_{0}\right)<\log r_{0}+C .
$$

Here $\rho=\log |x|+C$ is the equidistant hypersurface about the vertical geodesic line $\gamma$ connecting $p_{\infty}$ and $0 \in \mathbb{R}^{n} \subset \partial_{\infty} \mathbb{H}^{n+1}$. Then we claim

$$
\hat{\rho}(r) \leq \log r+C
$$

for all $r \in\left(r_{0}, R\right)$. Assume otherwise, there is some interval $\left[r_{1}, r_{2}\right] \subset\left(r_{0}, R\right)$, such that

$$
\hat{\rho}\left(r_{1}\right)=\log r_{1}+C \text { and } \hat{\rho}(r)>\log r+C \text { for } r \in\left(r_{1}, r_{2}\right] .
$$

Then there has to be some $\xi \in\left(r_{1}, r_{2}\right)$ where $\hat{\rho}$ is differentiable and $\hat{\rho}^{\prime}(\xi)>1 / \xi$. This implies the horizontal spherical section of $\hat{\Sigma}$ at $r=\xi$ has negative definite second fundamental form, in contrast to the equidistant hypersurface, whose horizontal spherical sections are totally geodesic. Because that $\hat{\Sigma}$ has nonnegative Ricci curvature when it is differentiable, and because the mean curvature of the spherical section only drops at singular point, one may derive that $\hat{\Sigma}$ is compact, which clearly is a contradiction. So we proved (6.3). To see $R=\infty$, we assume otherwise. Then, from the fact that $\Sigma$ is complete and has only one end at $p_{\infty}$, $\lim _{r \rightarrow R} \hat{\rho}(r)=\infty$, which contrdicts with (6.3). Hence $\Omega=\mathbb{R}^{n}$ and (6.3) holds for all $r \geq r_{0}$. Thus the proof of the lemma is completed.

Based on Theorems 3.1 and 4.2, we are able to improve the results on asymptotic behavior of global vertical graph of nonnegative sectional curvature in $[3,4]$. For the convenience of readers, we repeat our fourth main theorem in the introduction as follows:

Theorem 6.1 Suppose that $\Sigma$ is a properly embedded, complete hypersurface with nonnegative Ricci and single end. Then it is a global graph of $\rho=\rho(x)$ in Busemann coordinates and it is asymptotically rotationally symmetric in the sense that there is a number $m \in[0,1]$ such that

$$
m \log |x|+o(\log |x|) \leq \rho(x) \leq m \log |x|+C
$$

as $x \rightarrow \infty$ in $\mathbb{R}^{n}$. Moreover, $m=0$ implies that the hypersurface is a horosphere. In any case, the hypersurface $\Sigma$ always stays inside a horosphere and is supported by some equidistant hypersurface.

Proof As the first step, to use Theorem 3.1, we first want to change coordinates in hyperbolic space, that is, to choose a different point at infinity $\partial_{\infty} \mathbb{H}^{n+1}$ for the half space model. Then, 
based on Lemma 6.1, we know a priori that the hypersurface $\Sigma$ is below an equidistant hypersurface at least near the end at $p_{\infty}$. Hence, in the new Busemann coordinates, the hypersurface $\Sigma$ is no longer a global graph of the height function, rather, a graph of the height function over a punctured ball, $B(0, R) \backslash\{0\}$ in the new Busemann coordinates $(y, \tau)$ (in other words, we may put the end at infinity of $\Sigma$ at the origin of the new Busemann coordinates). Therefore we are looking at the part of the hypersurface $\Sigma$ that is parametrized as the graph of the height function $\tau=\tau(y)$, which is a $n$-subharmonic function in $B(0, R) \backslash\{0\}$ with $\lim _{y \rightarrow 0} \tau(y)=-\infty$. Thus, we may apply Theorem 3.1 to $-\tau$ and obtain

$$
\begin{gathered}
\tau(y) \leq-m_{1} \log \frac{1}{|y|}+C \text { for all } y \in B(0, R) \backslash\{0\} \\
\tau(y) \geq-m_{1} \log \frac{1}{|y|}+o\left(\log \frac{1}{|y|}\right) \text { for all } y \in(B(0, R) \backslash\{0\}) \backslash E
\end{gathered}
$$

for some $m_{1} \geq 1$ and a set $E$ that is $n$-thin at the origin. (6.4) can be improved to

$$
-m_{1} \log \frac{1}{|y|}+o\left(\log \frac{1}{|y|}\right) \leq \tau(y) \leq-m_{1} \log \frac{1}{|y|}+C \text { for all } y \in B(0, R) \backslash\{0\}
$$

Let us assume this temporarily.

For the convenience of readers, we have here the transformation laws of the change of parameterizations of hyperbolic space from ball model to half space model (cf. [53, Chapter 4]):

$$
\left\{\begin{array} { r l } 
{ y } & { = \frac { 2 z } { | Z - e _ { n + 1 } | ^ { 2 } } } \\
{ y _ { n + 1 } } & { = \frac { 1 - | Z | ^ { 2 } } { | Z - e _ { n + 1 } | ^ { 2 } } }
\end{array} \text { and } \left\{\begin{array}{rl}
x & =\frac{2 z}{\left|Z+e_{n+1}\right|^{2}} \\
x_{n+1} & =\frac{1-|Z|^{2}}{\left|Z+e_{n+1}\right|^{2}}
\end{array}\right.\right.
$$

for $Z=\left(z, z_{n+1}\right) \in B(0,1) \subset \mathbb{R}^{n+1}, Y=(y, y+n+1) \in \mathbb{R}_{+}^{n+1}$ and $X=\left(x, x_{n+1}\right) \in$ $\mathbb{R}_{+}^{n+1}$, where $e_{n+1}=(0,1)$ is the north pole of the unit sphere in $\mathbb{R}^{n+1}$. In $Y$ coordinates it takes the north pole to infinity and the south pole to the origin; while in $X$ coordinates it takes the south pole to infinity and the north pole to the origin. Hence the coordinate change between $X$ and $Y$ is the inversion with respect to the unit sphere centered at the origin:

$$
Y=\frac{X}{|X|^{2}} \quad \text { or }\left\{\begin{aligned}
y & =\frac{x}{|x|^{2}+x_{n+1}^{2}} \\
y_{n+1} & =\frac{x_{n+1}}{|x|^{2}+x_{n+1}^{2}} .
\end{aligned}\right.
$$

We may assume from the beginning that the hypersurface $\Sigma$ has its end at $p_{\infty}=-e_{n+1}$. Therefore

$$
\frac{1}{|y|}=|x| \cdot\left(1+\frac{x_{n+1}^{2}}{|x|^{2}}\right) \text { and } \tau=\log y_{n+1}=\rho-2 \log |x|-\log \left(1+\frac{x_{n+1}^{2}}{|x|^{2}}\right) .
$$

So we may translate (6.5) into

$$
m \log |x|+o(\log |x|) \leq \rho(x) \leq m \log |x|+C \text { for all } x \in \mathbb{R}^{n}
$$

for some $m=2-m_{1} \leq 1$. Here we use (6.2) from Lemma 6.1 to control $x_{n+1}^{2} /|x|^{2}$. 
Next step is to improve (6.4) and eliminate any nontrivial $n$-thin set $E$. Our approach here is to use the strict and global convexity of the hypersurface $\Sigma$ to rule out the nontrivial $n$-thin set $E$, which is close to that in $[3,4]$ in 2 dimensions but more straightforward. Assume otherwise, (6.5) is not true on a set $E$, which is $n$-thin and non-compact. Hence, there is a positive number $\epsilon_{0}$ and a sequence point $p_{k}=\left(s_{k} \theta_{k}, \tau\left(s_{k} \theta_{k}\right)\right) \in \Sigma$ such that

$$
y_{n+1}^{\Sigma}\left(s_{k} \theta_{k}\right)=e^{\tau\left(s_{k} \theta_{k}\right)}<s_{k}^{m_{1}+\epsilon_{0}}
$$

and $s_{k} \rightarrow 0$. We have, in the light of Lemma 5.1 and Definition 3.1, for each $s_{k} \theta_{k} \in E$, there always exists $\hat{s}_{k} \theta_{k} \notin E$ for $\hat{s}_{k} \in\left(s_{k}\left(1-s_{k}^{l}\right), s_{k}\right)$ for any fixed large $l \geq 1$. This can be proved by contradiction. Assume otherwise, one derives from (5.8) that, for each $i$,

$$
\operatorname{cap}_{n}\left(E \cap \omega_{i}, \Omega_{i}\right) \geq \frac{c_{n}}{(2 i(l+1) \log 2)^{n-1}},
$$

by the scaling invariance, which is impossible by Definition 3.1. On the other hand, there is $\delta_{0}$ such that

$$
y_{n+1}^{\Sigma}(s \theta)=e^{\tau(s \theta)} \geq s^{m_{1}+\frac{1}{2} \epsilon_{0}}
$$

for all $s \theta \notin E$ and $0<s<\delta_{0}$. In particular

$$
y_{n+1}^{\Sigma}\left(\hat{s}_{k} \theta_{k}\right) \geq \hat{s}_{k}^{m_{1}+\frac{1}{2} \epsilon_{0}} \geq a_{0} s_{k}^{m_{1}+\frac{1}{2} \epsilon_{0}}
$$

for some positive $a_{0}$, at least when $k$ is large. Let us assume the following is the equation for the semi-circle that is inside the hyperplane tangent to $\Sigma$ at the point over $\hat{s}_{k} \theta_{k}$ and in the 2-plane for the fixed $\theta_{k} \in \mathbb{S}^{n-1}$

$$
\left|s-c_{k}\right|^{2}+y_{n+1}^{2}=r_{k}^{2}=\left|\hat{s}_{k}-c_{k}\right|^{2}+\left(y_{n+1}^{\Sigma}\left(\hat{s}_{k} \theta_{k}\right)\right)^{2}
$$

where $\left(c_{k}, 0\right)$ is the center of the semi-circle and $0<c_{k}<s_{k}$ due to the fact that

$$
y_{n+1}^{\Sigma}\left(\hat{s}_{k} \theta_{k}\right)<y_{n+1}^{\Sigma}\left(s_{k} \theta_{k}\right) .
$$

We may estimate the height of this semi-circle at $s=s_{k}$ :

$$
\begin{aligned}
\left.y_{n+1}^{2}\right|_{s=s_{k}} & \geq a_{0}^{2} s_{k}^{2 m_{1}+\epsilon_{0}}+\left|\hat{s}_{k}-c_{k}\right|^{2}-\left|s_{k}-c_{k}\right|^{2} \\
& =a_{0}^{2} s_{k}^{2 m_{1}+\epsilon_{0}}+\left|\hat{s}_{k}-s_{k}\right|^{2}-2\left(s_{k}-\hat{s}_{k}\right) \cdot\left(s_{k}-c_{k}\right) \\
& \geq a_{0}^{2} s_{k}^{2 m_{1}+\epsilon_{0}}-c_{0} s_{k}^{2 m_{1}+1+2 \epsilon_{0}}>\left(y_{n+1}^{\Sigma}\left(s_{k} \theta_{k}\right)\right)^{2}
\end{aligned}
$$

for some uniform $a_{0}$ and $c_{0}$ and some appropriately large $l$, in the light of (6.7), which means the point $p_{k}$ on $\Sigma$ falls under the hyperplane and violates the strict and global convexity of $\Sigma$, at least when $k$ is large enough.

So far we have shown that

$$
m \log |x|+o(\log |x|) \leq \rho(x) \leq m \log |x|+C
$$

as $x \rightarrow \infty$ in $\mathbb{R}^{n}$ with $m \leq 1$. In the last step, we prove that $m \in[0,1]$ and $\Sigma$ is a horosphere when $m=0$. We at this point go back to the Busemann coordinates $(x, \rho)$, use the similar argument in the last step of the proof of Theorem 5.2 (even easier, because that there is no bad thin set), and obtain

$$
|m|^{n-2} m=\frac{1}{w_{n-1}} \int_{\mathbb{R}^{n}}\left(\Delta_{n} \rho\right) \mathrm{d} x \geq 0 .
$$


Therefore, when $m=0, \rho$ in fact is an $n$-harmonic function and upper bounded by a constant. In light of the Liouville Theorem [39, Theorem 6.2 and Corollary 6.11], $\rho$ is a constant, i.e. $\Sigma$ is a horosphere.

At last, it is easily seen that $\Sigma$ stays inside a horosphere, when $m>0$ or $m=0$, that is, there is some constant $C$ such that

$$
\rho\left(x_{1}, \ldots, x_{n}\right) \geq C \text {. }
$$

The fact that $\Sigma$ is supported by some equidistant hypersurface is proved in Lemma 6.1. The proof of Theorem 6.1 is completed.

Acknowledgements The first named author would like to thank Igor Verbitsky for helpful discussions. The authors are deeply grateful to the referee for thorough reading and numerous suggestions for improvements in writing.

Open Access This article is licensed under a Creative Commons Attribution 4.0 International License, which permits use, sharing, adaptation, distribution and reproduction in any medium or format, as long as you give appropriate credit to the original author(s) and the source, provide a link to the Creative Commons licence, and indicate if changes were made. The images or other third party material in this article are included in the article's Creative Commons licence, unless indicated otherwise in a credit line to the material. If material is not included in the article's Creative Commons licence and your intended use is not permitted by statutory regulation or exceeds the permitted use, you will need to obtain permission directly from the copyright holder. To view a copy of this licence, visit http://creativecommons.org/licenses/by/4.0/.

\section{References}

1. Adams, D.R., Hedberg, L.I.: Function Spaces and Potential Theory. Springer-Verlag, Berlin Heidelberg (1996)

2. Adams, D., Meyers, N.G.: Thinness and Wiener criteria for non-linear potentials. Indiana Univ. Math. J. 22, 169-197 (1972)

3. Alexander, S., Currier, R.J.: Non-negatively curved hypersurfaces of hyperbolic space and subharmonic functions. J. Lond. Math. Soc. 41(2), 347-360 (1990)

4. Alexander, S., Currier, R.J.: Hypersurfaces and nonnegative curvature. Proc. Symposia Pure Math. 54(3), 37-44 (1993)

5. Arsove, M.: Functions of potential type. Trans. AMS 75, 526-551 (1953)

6. Arsove, M., Huber, A.: Local behavior of subharmonic functions. Indiana Univ. Math. J. 22, 1191-1199 (1973)

7. Bando, S., Kasue, A., Nakajima, H.: On a construction of coordinates at infinity on manifolds with fast curvature decay and maximal volume growth. Invent. Math. 97, 313-349 (1989)

8. Boccardo, L., Gallouët, T.: Nonlinear elliptic equations with right hand side measures. Commun. Part. Differ. Equ. 17, 641-655 (1992)

9. Bidaut-Veron, M.F.: Local and global behavior of solutions of quasi- linear equations of Emden-fowler type. Arch. Ration. Mech. Anal. 107(4), 293-324 (1989)

10. Blanc, C., Fiala, F.: le type d'une surface et sa courbure totale. Comment. Math. Helv. 14, 230-233 $(1941-42)$

11. Bonini, V., Ma, S., Qing, J.: On non-negatively curved hypersurfaces in hyperbolic space, to appear in Mathematische Annalen. arXiv: 1603.03862

12. Bonini, V., Ma, S., Qing, J.: Hypersurfaces with nonnegative Ricci curvature in hyperbolic space, preprint 2017. arXiv: 1709.00091

13. Brelot, M.: Points irreguliers et transformations continues en thëorie du potentiel. J. de Math. 19, 319-337 (1940)

14. Brelot, M.: Sur les ensembles effilés. Bull. Sci. Math. 68, 12-36 (1944)

15. Brezis, H., Merle, F.: Uniform estimates and blow-up behavior for solutions of $-\Delta u=V(x) e^{u}$ in two dimensions. Commun. PDE 16, 1223-1253 (1991)

16. Bromwich, T.: Introduction to the Theory of Infinite Series. The Macmillan, New York (1908)

17. Carron, G., Herzlich, M.: The Huber theorem for non-compact conformally flat manifolds. Comment. Math. Helv. 77, 192-220 (2002) 
18. Carron, G., Herzlich, M.: Conformally flat manifolds with nonnegative Ricci curvature. Compos. Math. 142(3), 798-810 (2006)

19. Chang, S.-Y.A., Eastwood, M., Ørsted, B., Yang, P.: What is Q-curvature? Acta Appl. Math. 102(2-3), 119-125 (2008)

20. Chang, S.-Y.A., Hang, F.B., Yang, P.: On a class of locally conformally flat manifolds. IMRN 4, 185-209 (2004)

21. Chang, S.-Y.A., Gursky, M., Yang, P.: An equation of Monge-Ampére type in conformal geometry, and four-manifolds of positive Ricci curvature. Ann. of Math. (2) 155(3), 709-787 (2002)

22. Chang, S.-Y.A., Qing, J., Yang, P.: Compactification of a class of conformally flat 4-manifold. Invent. Math. 142, 65-93 (2000)

23. Chen, B., Zhu, X.: A gap theorem for complete noncompact manifolds with nonnegative Ricci curvature. Commun. Geom. Anal. 10(1), 217-239 (2002)

24. Cohn-Vossen, S.: Kürzeste Wege und Totalkrummung auf Flächen. Compositio Math. 2, 69-133 (1935)

25. Colding, T.H.: Ricci curvature and volume convergence. Ann. Math. 145, 477-501 (1997)

26. Croke, C.B., Karcher, H.: Volume of small balls on open manifolds: lower bounds and examples. Trans. Am. Math. Soc. 309(2), 753-762 (1988)

27. Dolzmann, G., Hungerbühler, N., Müller, S.: Non-linear elliptic systems with measure-valued right hand side. Math. Z. 226, 545-574 (1997)

28. Epstein, C.L.: The hyperbolic Gauss map and quasi-conformal reflections. J. Reine Angew. Math. 372, 96-135 (1986)

29. Epstein, C.L.: Envelopes of horospheres and Weingarten surfaces in hyperbolic 3-space, Unpublished (1986). http://www.math.upenn.edu/ cle/papers/index.html

30. Epstein, C.L.: The asymptotic boundary of a surface imbedded in $\mathbb{H}^{3}$ with nonnegative curvature. Mich. Math. J. 34, 227-239 (1987)

31. Fiala, F.: Le probléme des isopérimétres sur les surfaces ouvertes á courbure positive. Comment. Math. Helv. 13, 293-346 (1940-41)

32. Fontana, L., Morpurgo, C.: Adams inequalities on measure spaces. Adv. Math. 226(6), 5066-5119 (2011)

33. Gehring, F.W.: Symmetrization of rings in space. Trans. Am. Math. Soc. 101, 499-519 (1961)

34. González, M.: Singular sets of a class of locally conformally flat manifolds. Duke Math. J. 129(3), 551-572 (2005)

35. Guan, P., Lin, C., Wang, G.: Schouten tensor and some topological properties. Commun. Anal. Geom. 13(5), 887-902 (2005)

36. Hayman, W.K.: Slowly growing integral and subharmonic functions. Comment. Math. Helv. 34, 75-84 (1960)

37. Hayman, W.K., Kennedy, P.B.: Subharmonic Functions, vol. 1. Academic Press, London, New York, San Francisco (1976)

38. Heinonen, J., Kilpeläinen, T.: On the Wiener criterion and quasilinear obstacle problems. Trans. Am. Math. Soc. 310, 239-255 (1988)

39. Heinonen, J., Kilpeläinen, T., Martio, O.: Nonlinear Potential Theory of Degenerate Elliptic Equations. Oxford Univ. Press, Oxford (1993)

40. Heins, M.H.: Entire functions with bounded minimum modulus; subharmonic function analogous. Ann. Math. 49, 200-213 (1948)

41. Hopf, H.: Sutta geometria globale délie superficie. Rendiconti del Seminario Matematico e Fisico di Milano 23, 48-63 (1952)

42. Huber, A.: Über Wachstumseigenschaften gewisser Klaussen von subharmonischen Funktionen. Comment. Math. Helv. 26, 81-116 (1952)

43. Huber, A.: On subharmonic functions and differential geometry in the large. Comment. Math. Helv. 32, 13-72 (1957)

44. Ioku, N.: Brezis-Merle type inequality for a weak solution to n-Laplace equation in Lorentz-Zygmond space. Differ. Integr. Equ. 22(5-6), 459-518 (2009)

45. John, F., Nirenberg, L.: On functions of bounded mean oscillation. Commun. Pure Appl. Math. 14, 415-426 (1961)

46. Kilpeläinen, T., Malý, J.: $\mathcal{A}$-superharmonic functions and supersolutious of degenerate elliptic equations. Ark. Mat. 26, 87-105 (1988)

47. Kilpeläinen, T., Malý, J.: Degenerate elliptic equations with measure data and nonlinear potentials. Ann. Scuola Norm. Sup. Pisa Cl. Sci. (4) 19(4), 591-613 (1992)

48. Kilpeläinen, T., Malý, J.: The wiener test and potential estimates for quasilinear elliptic equations. Acta. Math. 172, 137-161 (1994)

49. Kichenassamy, S., Veron, L.: Singular solutions of the p-Laplace equation. Math. Ann. 275, 599-615 (1986) 
50. Kichenassamy, S., Veron, L.: Erratum: "Singular solutions of the p-Laplace equation" [Math. Ann. 275(4), 599-615 (1986)]. Math. Ann. 277(2), 352 (1987)

51. Lindqvist, P.: Notes on the p-Laplace Equation. University of Jyvaskyla, Lecture Notes (2006)

52. Phuc, N., Verbitsky, I.: Quasilinear and hessian equations of Lane-Emden type. Ann. Math. 168, 859-914 (2008)

53. Ratcliffe, J.G.: Foundations of Hyperbolic Manifolds. Graduate Texts in Mathematics, vol. 149. SpringerVerlag, New York (1994)

54. Rakotoson, J.: Quasilinear elliptic problems with measures as data. Differ. Integr. Equ. 4(3), 449-457 (1991)

55. Reshetnyak, Y.G.: Mappings with bounded deformation as extremals of Dirichlet type integrals. Sibirsk. Mat. Zh. 9, 652-666 (1966)

56. Reshetnyak, Y.G.: Reshetnyak. Stability theorems in geometry and analysis. Mathematics and Its Applications, Yurii (1994)

57. du Bois-Reymond, P.: Eine neue Theorie der Convergenz und Divergenz von Reihen mit positiven Gliedern. Journal für die reine und angewandte Mathematik 76, 61-91 (1873)

58. Schoen, R., Yau, S.T.: Conformally flat manifolds, Kleinian groups and scalar curvature. Invent. Math. 92, 47-71 (1988)

59. Serrin, J.: Local behavior of solutions of quasilinear equations. Acta Math. 111, 247-302 (1964)

60. Serrin, J.: Isolated singularities of solutions of quasilinear equations. Acta Math. 113, 219-240 (1965)

61. Taliaferro, S.: On the growth of superharmonic functions near an isolated singularity I. J. Differ. Equ. 158, 28-47 (1999)

62. Taliaferro, S.: Isolated singularities of nonlinear elliptic inequalities. Indiana Univ. Math. J. 50(4), 18851897 (2001)

63. Taliaferro, S.: Isolated singularities of nonlinear elliptic inequalities. II. Asymptotic behavior of solutions. Indiana Univ. Math. J. 55(6), 1791-1811 (2006)

64. Tolksdorf, P.: On the Dirichlet problem for quasilinear equations in domain with conical boundary points. Commun. Part. Differ. Equ. 8, 773-817 (1983)

65. Veron, L.: Local and Global Aspects of Quasilinear Degenerate Elliptic Equations. World Scientific, New Jersey (2017)

66. Zheng, S.: A strong convergence of the weak gradient to $\mathcal{A}$-harmonic type operators with $L^{1}$ data. J. Math. Anal. Appl. 430, 381-389 (2015)

67. Zhu, S.: The classification of complete locally conformally flat manifolds of nonnegative Ricci curvature. Pac. J. Math. 163(1), 189-199 (1994)

Publisher's Note Springer Nature remains neutral with regard to jurisdictional claims in published maps and institutional affiliations. 